\title{
Orlicz-Aleksandrov-Fenchel Inequality for Orlicz Multiple Mixed Volumes
}

\author{
Chang-Jian Zhao \\ Department of Mathematics, China Jiliang University, Hangzhou 310018, Zhejiang, China \\ Correspondence should be addressed to Chang-Jian Zhao; chjzhao@163.com
}

Received 4 May 2018; Revised 9 August 2018; Accepted 14 August 2018; Published 16 September 2018

Academic Editor: Alberto Fiorenza

Copyright (C) 2018 Chang-Jian Zhao. This is an open access article distributed under the Creative Commons Attribution License, which permits unrestricted use, distribution, and reproduction in any medium, provided the original work is properly cited.

Our main aim is to generalize the classical mixed volume $V\left(K_{1}, \ldots, K_{n}\right)$ and Aleksandrov-Fenchel inequality to the Orlicz space. In the framework of Orlicz-Brunn-Minkowski theory, we introduce a new affine geometric quantity by calculating the Orlicz firstorder variation of the mixed volume and call it Orlicz multiple mixed volume of convex bodies $K_{1}, \ldots, K_{n}$, and $L_{n}$, denoted by $V_{\varphi}\left(K_{1}, \ldots, K_{n}, L_{n}\right)$, which involves $(n+1)$ convex bodies in $\mathbb{R}^{n}$. The fundamental notions and conclusions of the mixed volume and Aleksandrov-Fenchel inequality are extended to an Orlicz setting. The related concepts and inequalities of $L_{p}$-multiple mixed volume $V_{p}\left(K_{1}, \ldots, K_{n}, L_{n}\right)$ are also derived. The Orlicz-Aleksandrov-Fenchel inequality in special cases yields $L_{p}$-AleksandrovFenchel inequality, Orlicz-Minkowski inequality, and Orlicz isoperimetric type inequalities. As application, a new Orlicz-BrunnMinkowski inequality for Orlicz harmonic addition is established, which implies Orlicz-Brunn-Minkowski inequalities for the volumes and quermassintegrals.

\section{Introduction}

One of the most important operations in geometry is vector addition. As an operation between sets $K$ and $L$, defined by

$$
K+L=\{x+y: x \in K, y \in L\},
$$

it is usually called Minkowski addition, and combined with volume, plays an important role in the Brunn-Minkowski theory. During the last few decades, the theory has been extended to $L_{p}$-Brunn-Minkowski theory. The set, called $L_{p}$ addition, was introduced by Firey in $[1,2]$. The operation, denoted by ${ }_{p}$, is defined by

$$
h\left(K+{ }_{p} L, x\right)^{p}=h(K, x)^{p}+h(L, x)^{p},
$$

for all $x \in \mathbb{R}^{n}, 1 \leq p \leq \infty$, and compact convex sets $K$ and $L$ in $\mathbb{R}^{n}$ containing the origin. When $p=\infty$, (2) is interpreted as $h\left(K+_{\infty} L, x\right)=\max \{h(K, x), h(L, x)\}$, as is customary. Here the functions are the support functions. If $K$ is a nonempty closed (not necessarily bounded) convex set in $\mathbb{R}^{n}$, then

$$
h(K, x)=\max \{x \cdot y: y \in K\},
$$

for $x \in \mathbb{R}^{n}$, defines the support function $h(K, x)$ of $K . L_{p}$ addition, volume, and combine inequalities lead to the $L_{p^{-}}$Brunn-Minkowski theory, which are the fundamental and core content in the $L_{p}$ Brunn-Minkowski theory. For some important results and more information from this theory, we refer to [3-22] and the references therein.

$L_{p}$ spaces have a natural generalization, known as the Orlicz spaces. In recent years, progress towards an OrliczBrunn-Minkowski theory is initiated by Lutwak, Yang, and Zhang $[23,24]$ to initiate an extension of the $L_{p}$ BrunnMinkowski theory to an Orlicz-Brunn-Minkowski theory. They successively established the fundamental affine inequalities for these bodies. The definition of a corresponding addition is shown in later work of Gardner, Hug, and Weil [25] and they developed a very general and comprehensive Orlicz-Brunn-Minkowski theory. And they constructed a general framework for the Orlicz-Brunn-Minkowski theory and made clear for the first time the relation to Orlicz spaces and norms. The Orlicz addition of convex bodies was also introduced from different angles and established the OrliczBrunn-Minkowski inequality for the Orlicz addition (see [26]). The Orlicz centroid inequality for star bodies was introduced which is an extension from convex to star bodies 
(see [27]). The existence of even Orlicz-Minkowski problem is demonstrated by Haberl, Lutwak, Yang, and Zhang [28]. Ludwig [29], Ludwig, and Reitzner [11] introduced what soon came to be seen as the Orlicz affine area; for related work, see [30]. In these papers the notions of $L_{p}$-addition, $L_{p}$-mixed volume, $L_{p}$-affine surface area, $L_{p}$-centroid body, and $L_{p^{-}}$ projection body and inequalities were extended to an Orlicz setting. Advances in the theory and its dual can be found in [31-48].

In 2014, Gardner, Hug, and Weil [25] introduced the Orlicz addition $K+{ }_{\varphi} L$ of compact convex sets $K$ and $L$ in $\mathbb{R}^{n}$ containing the origin, implicitly, by

$$
\begin{aligned}
& h\left(K+{ }_{\varphi} L, u\right) \\
& \quad=\inf \left\{\lambda>0: \varphi\left(\frac{h(K, u)}{\lambda}\right)+\varphi\left(\frac{h(L, u)}{\lambda}\right) \leq 1\right\},
\end{aligned}
$$

where $\varphi:[0, \infty) \longrightarrow(0, \infty)$ is a convex and increasing function such that $\varphi(0)=0$ and $\varphi(1)=1$. Let $\Phi$ denote the set of convex functions $\varphi:[0, \infty) \longrightarrow[0, \infty)$ that is increasing and satisfies $\varphi(0)=0$ and $\varphi(1)=1$. For $\varphi(t)=t^{p}$ and $p \geq 1$, the new addition $K+{ }_{\varphi} L$ becomes $K+{ }_{p} L$. The Orlicz mixed volume with respect to the Orlicz addition, denoted by $V_{\varphi}(K, L)$, is defined by

$$
V_{\varphi}(K, L)=\frac{1}{n} \int_{S^{n-1}} \varphi\left(\frac{h(L, u)}{h(K, u)}\right) h(K, u) d S(K ; u),
$$

where $S(K ; u)$ is the surface area measure of $K, K$ is a convex body containing the origin in its interior, and $L$ is a compact convex set containing the origin. For $\varphi(t)=t^{p}$ and $p \geq 1$, $V_{\varphi}(K, L)$ becomes $V_{p}(K, L)$, defined by (see [49])

$$
V_{p}(K, L)=\frac{1}{n} \int_{S^{n-1}} h(L, u)^{p} h(K, u)^{1-p} d S(K ; u) .
$$

Associated with the convex bodies $K_{1}, \ldots, K_{n-1}$ in $\mathbb{R}^{n}$ is a unique positive Borel measure on $S^{n-1}, S\left(K_{1}, \ldots, K_{n-1} ; \cdot\right)$; call it the mixed area measure of $K_{1}, \ldots, K_{n-1}$. For any convex body $K_{n}$, one has the integral representation (see, e.g., [5], p. 354).

$$
\begin{aligned}
V & \left(K_{1}, \ldots, K_{n}\right) \\
\quad & =\frac{1}{n} \int_{S^{n-1}} h\left(K_{n}, u\right) d S\left(K_{1}, \ldots, K_{n-1} ; u\right) .
\end{aligned}
$$

The integration is with respect to the mixed area measure $S\left(K_{1}, \ldots, K_{n-1} ; \cdot\right)$ on $S^{n-1}$. The mixed area measure $S\left(K_{1}, \ldots, K_{n-1} ; \cdot\right)$ is symmetric in its (first $\left.n-1\right)$ arguments. When $K_{1}=\cdots=K_{n-i-1}=K$ and $K_{n-i}=\cdots=K_{n-1}=B$, the mixed area measure $S(K, \ldots, K, B, \ldots, B ; \cdot)$ with $i$ copies of $B$ and $(n-i-1)$ copies of $K$ will be written as $S_{i}(K, \cdot)$. For $K_{1}=\cdots=K_{n-1}=K, S\left(K_{1}, \ldots, K_{n-1} ; \cdot\right)$ reduces to the surface area measure $S(K ; \cdot)$. The classical AleksandrovFenchel inequality is as follows: if $K_{1}, \ldots, K_{n}$ are convex bodies containing the origin and $1 \leq r \leq n$ (see, e.g., [50, p. 401]), then

$$
V\left(K_{1}, \ldots, K_{n}\right) \geq \prod_{i=1}^{r} V\left(K_{i}, \ldots, K_{i}, K_{r+1}, \ldots, K_{n}\right)^{1 / r} .
$$

In the paper, we further improve the Orlicz-BrunnMinkowski theory. Our main aim is to introduce a new mixed volume of convex bodies $K_{1}, \ldots, K_{n}, L_{n}$, denoted as $V_{\varphi}\left(K_{1}, \ldots, K_{n}, L_{n}\right)$, which involves $(n+1)$ convex bodies in $\mathbb{R}^{n}$, and call it Orlicz multiple mixed volume for convex bodies $K_{1}, \ldots, K_{n}$, and $L_{n}$. The fundamental notions and conclusions of the mixed volume and Aleksandrov-Fenchel inequality are extended to an Orlicz setting. The new OrliczAleksandrov-Fenchel inequality in special cases yields $L_{p^{-}}$ Aleksandrov-Fenchel inequality, Orlicz-Minkowski inequality, and Orlicz isoperimetric type inequality. As application, we prove Orlicz-Brunn-Minkowski inequality for the mixed volumes, which implies the Orlicz-Brunn-Minkowski inequalities for the volumes and quermassintegrals.

Following the spirit of introduction of Aleksandrov [51], Fenchel and Jessen's mixed quermassintegrals (see [52]), and introduction of Lutwak's $L_{p}$-mixed quermassintegrals (see [49]), we are based on the study of Orlicz first-order variational of mixed volumes. In Section 4, we prove that the Orlicz first-order variation of mixed volumes can be expressed as follows: for $\varphi \in \Phi$ and $\varepsilon>0$

$$
\begin{aligned}
& \left.\frac{d}{d \varepsilon}\right|_{\varepsilon=0^{+}} V\left(K_{1}, \ldots, K_{n-1}, L_{n}{ }_{\varphi, \varepsilon} K_{n}\right) \\
& \quad=\frac{1}{\varphi_{l}^{\prime}(1)} \cdot V_{\varphi}\left(K_{1}, \ldots, K_{n}, L_{n}\right),
\end{aligned}
$$

where $K_{1}, \ldots, K_{n}$ are convex bodies containing the origin, $L_{n}$ is a convex body containing the origin in its interior, $V\left(K_{1}, \ldots, K_{n}\right)$ is the usual mixed volume, and $\varphi_{l}^{\prime}(1)$ denotes the value of left derivative of convex function $\varphi$ at point 1 . Here, $K+{ }_{\varphi, \varepsilon} L$ denotes the Orlicz linear combination of convex bodies $K$ and $L$. If $K, L$ are convex bodies containing the origin, $\alpha, \beta \geq 0$, and $\varphi \in \Phi$, then Orlicz linear combination ${ }^{+}(K, L, \alpha, \beta)$ is defined by $([25])$

$$
\begin{gathered}
\alpha \cdot \varphi\left(\frac{h(K, x)}{h\left(+_{\varphi}(K, L, \alpha, \beta), x\right)}\right)+\beta \\
\cdot \varphi\left(\frac{h(L, x)}{h\left(+_{\varphi}(K, L, \alpha, \beta), x\right)}\right)=1 .
\end{gathered}
$$

For $\alpha=1$ and $\beta=\varepsilon \geq 0$, the Orlicz linear combination $+_{\varphi}(K, L, 1, \varepsilon)$ is denoted by $K+{ }_{\varphi, \varepsilon} L$. This first-order variational equation then provides the new geometric quantity $V_{\varphi}\left(K_{1}, \ldots, K_{n}, L_{n}\right)$; it is called it Orlicz multiple mixed volume of convex bodies $K_{1}, \ldots, K_{n}, L_{n}$ and defined by

$$
\begin{aligned}
& V_{\varphi}\left(K_{1}, \ldots, K_{n}, L_{n}\right) \\
& \quad:=\varphi_{l}^{\prime}(1) \cdot\left(\left.\frac{d}{d \varepsilon}\right|_{\varepsilon=0^{+}} V\left(K_{1}, \ldots, K_{n-1}, L_{n}{ }_{\varphi, \varepsilon} K_{n}\right)\right) .
\end{aligned}
$$

We prove also the new affine geometric quantity $V_{\varphi}\left(K_{1}, \ldots\right.$, $K_{n}, L_{n}$ ) which has an integral representation. 


$$
\begin{aligned}
& V_{\varphi}\left(K_{1}, \ldots, K_{n}, L_{n}\right)=\frac{1}{n} \\
& \cdot \int_{S^{n-1}} \varphi\left(\frac{h\left(K_{n}, u\right)}{h\left(L_{n}, u\right)}\right) h\left(L_{n}, u\right) \\
& \cdot d S\left(K_{1}, \ldots, K_{n-1} ; u\right) .
\end{aligned}
$$

When $\varphi(t)=t^{p}$ and $p \geq 1, V_{\varphi}\left(K_{1}, \ldots, K_{n}, L_{n}\right)$ becomes a new mixed volume $V_{p}\left(K_{1}, \ldots, K_{n}, L_{n}\right)$; call it $L_{p}$-multiple mixed volume, which is first published and named here.

$$
\begin{aligned}
& V_{p}\left(K_{1}, \ldots, K_{n}, L_{n}\right)=\frac{1}{n} \\
& \quad \cdot \int_{S^{n-1}} h\left(K_{n}, u\right)^{p} h\left(L_{n}, u\right)^{1-p} d S\left(K_{1}, \ldots, K_{n-1} ; u\right) .
\end{aligned}
$$

For $p=1, V_{p}\left(K_{1}, \ldots, K_{n}, L_{n}\right)$ becomes $V\left(K_{1}, \ldots, K_{n}\right)$, and for $K_{1}=\cdots=K_{n-i-1}=K, K_{n-i}=\cdots=K_{n-1}=B$, $K_{n}=L$ and $L_{n}=K, V_{p}\left(K_{1}, \ldots, K_{n}, L_{n}\right)$ becomes the $p$-mixed quermassintegral $W_{p, i}(K, L)$ of $K$ and $L$, defined by, for all $0 \leq i<n$ (see [49]),

$$
W_{p, i}(K, L)=\frac{1}{n} \int_{S^{n-1}} h(L, u)^{p} h(K, u)^{1-p} d S_{i}(K ; u) .
$$

Putting $K_{n}=L, L_{n}=K$, and $K_{1}=\cdots=K_{n-1}=$ $K$ in (12), $V_{\varphi}\left(K_{1}, \ldots, K_{n}, L_{n}\right)$ becomes the well-know Orlicz mixed volume $V_{\varphi}(K, L)$. This shows that the well-known mixed volumes $V_{p}(K, L), W_{p, i}(K, L)$, and $V_{\varphi}(K, L)$ are all the special cases of $V_{\varphi}\left(K_{1}, \ldots, K_{n}, L_{n}\right)$, where $p \geq 1$ and $\varphi \in$ $\Phi$. In fact, the Orlicz mixed quermassintegral $W_{\varphi, i}(K, L)$ is also a special case of $V_{\varphi}\left(K_{1}, \ldots, K_{n}, L_{n}\right)$. The Orlicz mixed quermassintegral of $K$ and $L, W_{\varphi, i}(K, L)$, is defined by (see Section 4)

$$
W_{\varphi, i}(K, L)=\frac{1}{n} \int_{S^{n-1}} \varphi\left(\frac{h(L, u)}{h(K, u)}\right) h(K, u) d S_{i}(K, u) .
$$

In Section 5, we establish the following OrliczAleksandrov-Fenchel inequality for the Orlicz multiple mixed volume. If $K_{1}, \ldots, K_{n}$ are convex bodies containing the origin, $L_{n}$ is a convex body containing the origin in its interior, $1 \leq r \leq n$, and $\varphi \in \Phi$, then

$$
\begin{aligned}
V_{\varphi}( & \left.K_{1}, \ldots, K_{n}, L_{n}\right) \\
\geq & V\left(K_{1}, \ldots, K_{n-1}, L_{n}\right) \\
& \quad \cdot \varphi\left(\frac{\prod_{i=1}^{r} V\left(K_{i}, \ldots, K_{i}, K_{r+1}, \ldots, K_{n}\right)^{1 / r}}{V\left(K_{1}, \ldots, K_{n-1}, L_{n}\right)}\right) .
\end{aligned}
$$

When $\varphi(t)=t^{p}$ and $p \geq 1$, (16) becomes a new $L_{p^{-}}$ Aleksandrov-Fenchel type inequality. If $K_{1}, \ldots, K_{n}$ are convex bodies containing the origin, $L_{n}$ is a convex body containing the origin in its interior, $1 \leq r \leq n$, and $p \geq 1$, then

$$
\begin{aligned}
V_{p} & \left(K_{1}, \ldots, K_{n}, L_{n}\right) \\
& \geq \frac{\prod_{i=1}^{r} V\left(K_{i}, \ldots, K_{i}, K_{r+1}, \ldots, K_{n}\right)^{p / r}}{V\left(K_{1}, \ldots, K_{n-1}, L_{n}\right)^{p-1}} .
\end{aligned}
$$

This $L_{p}$ type inequality has never been discovered before, which is first published and named here. Obviously, for $p=1$, (17) becomes (8). When $r=n, K_{1}=\cdots=K_{n-1}=K, K_{n}=L$, and $L_{n}=K$, (16) becomes the following inequality which was established by Gardner, Hug, and Weil [25]. If $K$ is a convex body containing the origin in its interior, $L$ is a convex body containing the origin, and $\varphi \in \Phi$, then

$$
V_{\varphi}(K, L) \geq V(K) \cdot \varphi\left(\left(\frac{V(L)}{V(K)}\right)^{1 / n}\right)
$$

If $\varphi$ is strictly convex, equality holds if and only if $K$ and $L$ are homothetic. In Section 5, we show also that (16) in special case yields the following result. If $K$ is a convex body containing the origin in its interior, $L$ is a convex body containing the origin, $0 \leq i<n$, and $\varphi \in \Phi$, then (see [43])

$$
W_{\varphi, i}(K, L) \geq W_{i}(K) \cdot \varphi\left(\left(\frac{W_{i}(L)}{W_{i}(K)}\right)^{1 /(n-i)}\right)
$$

If $\varphi$ is strictly convex, equality holds if and only if $K$ and $L$ are homothetic, where $W_{i}(K)$ is the usual quermassintegral of convex body $K$.

The classical isoperimetric inequality states that if $K$ is convex body (see, e.g., [50, p.382]), then

$$
\left(\frac{S(K)}{\omega_{n}}\right)^{n} \geq\left(\frac{V(K)}{\kappa_{n}}\right)^{n-1}
$$

with equality if and only if $K$ is ball, where $S(K)$ is the surface area of $K$ and $\kappa_{n}$ denotes volume of the unit ball $B$ and its surface area by $\omega_{n}$. The Orlicz isoperimetric inequality is established in Section 5. If $K$ is a convex body containing the origin, $\varphi \in \Phi$, and $0 \leq i<n$, then

$$
\begin{gathered}
\frac{V_{\varphi}(K[n-i-1], B[i+1], K)}{W_{i}(K)} \\
\geq \varphi\left(\left(\frac{\kappa_{n}}{W_{i}(K)}\right)^{1 /(n-i)}\right) .
\end{gathered}
$$

If $\varphi$ is strictly convex, equality holds if and only if $K$ is a ball, where $V_{\varphi}(K[n-i-1], B[i+1], K)$ denotes the Orlicz multiple mixed volume $V_{\varphi}(\underbrace{K, \ldots, K}_{n-i-1}, \underbrace{B, \ldots, B}_{i+1}, K)$. When $\varphi(t)=t^{p}$ and $p \geq 1,(21)$ becomes the following $L_{p}$-isoperimetric inequality. If $K$ is a convex body containing the origin, $p \geq 1$, and $0 \leq i<n$, then

$$
\left(\frac{n W_{p, i}(K, B)}{\omega_{n}}\right)^{n-i} \geq\left(\frac{W_{i}(K)}{\kappa_{n}}\right)^{n-i-p}
$$

with equality if and only if $K$ is a ball, where $W_{p, i}(K, B)$ denotes the mixed $p$-quermassintegral of $K$ and $B$. For $i=0$ and $p=1$, (22) becomes isoperimetric inequality (20). 
In Section 6, we establish the following Orlicz-BrunnMinkowski type inequality. If $K_{1}, \ldots, K_{n}, L_{n}$ are convex bodies containing the origin and $\varphi \in \Phi$, then for $\varepsilon>0$

$$
\begin{gathered}
1 \geq \varphi\left(\frac{V\left(K_{1}, \ldots, K_{n}\right)}{V\left(K_{1}, \ldots, K_{n-1}, K_{n}{ }^{+}{ }_{\varphi, \varepsilon} L_{n}\right)}\right)+\varepsilon \\
\cdot \varphi\left(\frac{V\left(K_{1}, \ldots, K_{n-1}, L_{n}\right)}{V\left(K_{1}, \ldots, K_{n-1}, K_{n}{ }_{\varphi, \varepsilon} L_{n}\right)}\right),
\end{gathered}
$$

If $\varphi$ is strictly convex, equality holds if and only if $K_{n}$ and $L_{n}$ are homothetic. Inequality (23) in special case yields the following inequality, which was established by Gardner, Hug, and Weil [25]. If $K, L$ are convex bodies containing the origin and $\varphi \in \Phi$

$$
\begin{aligned}
& 1 \geq \varphi\left(\left(\frac{V(K)}{V\left(K+_{\varphi} L\right)}\right)^{1 / n}\right) \\
& +\varphi\left(\left(\frac{V(L)}{V\left(K+_{\varphi} L\right)}\right)^{1 / n}\right) .
\end{aligned}
$$

If $\varphi$ is strictly convex, equality holds if and only if $K$ and $L$ are homothetic (also see [26]). In fact, the following inequality is also the special case of (23). If $K, L$ are convex bodies containing the origin, $\varphi \in \Phi$, and $0 \leq i<n$, then (see [43])

$$
\begin{aligned}
1 \geq & \varphi\left(\left(\frac{W_{i}(K)}{W_{i}\left(K+_{\varphi} L\right)}\right)^{1 /(n-i)}\right) \\
& +\varphi\left(\left(\frac{W_{i}(L)}{W_{i}\left(K+{ }_{\varphi} L\right)}\right)^{1 /(n-i)}\right) .
\end{aligned}
$$

If $\varphi$ is strictly convex, equality holds if and only if $K$ and $L$ are homothetic.

This paper is organized as follows. In Section 2, we collect some basic concepts and facts that will be used in the proofs of our results. The Orlicz addition and Orlicz linear combination are introduced in Section 3. In Section 4, we introduce the Orlicz multiple mixed volumes, and some of its basic properties and lemmas are shown and hence $L_{p}$-multiple mixed volume $V_{p}\left(K_{1}, \ldots, K_{n}, L_{n}\right)$ of convex bodies $K_{1}, \ldots, K_{n}, L_{n}$ is also derived. In Section 5, Orlicz-Aleksandrov-Fenchel inequality is established, which in special case yields an $L_{p}$-Aleksandrov-Fenchel inequality and Orlicz-Minkowski inequality, respectively. The Orlicz isoperimetric and Urysohn's inequalities are given, which imply the $L_{p}$-isoperimetric inequality and $L_{p}$-Urysohn's inequality, respectively. In Section 6, a new Orlicz-BrunnMinkowski inequality for the Orlicz addition is established, which is a generalization of the Orlicz-Brunn-Minkowski inequality for volumes and quermassintegrals.

\section{Notations and Preliminaries}

The setting for this paper is $n$-dimensional Euclidean space $\mathbb{R}^{n}$. Let $\mathscr{K}^{n}$ denote the set of convex bodies (compact convex subsets with nonempty interiors) in $\mathbb{R}^{n}$, let $\mathscr{K}_{o}^{n}$ be the class of members of $\mathscr{K}^{n}$ containing the origin, and let $\mathscr{K}_{\text {oo }}^{n}$ be those sets in $\mathscr{K}^{n}$ containing the origin in their interiors. We reserve the letter $u \in S^{n-1}$ for unit vectors. $S^{n-1}$ is the unit sphere. For a compact set $K$, we write $V(K)$ for the $(n$ dimensional) Lebesgue measure of $K$ and call this the volume of $K$. A nonempty closed convex set is uniquely determined by its support function. Support function is homogeneous of degree 1; that is,

$$
h(K, r x)=r h(K, x),
$$

for all $x \in \mathbb{R}^{n}$ and $r \geq 0$. Obviously, for a pair of compact convex sets $K$ and $L$, we have

$$
h(K, x) \leq h(L, x), \quad \text { if and only if } K \subset L .
$$

A function is a support function of a compact convex set if and only if it is positively homogeneous degree one and subadditive. Let $d$ denote the Hausdorff metric on $\mathscr{K}^{n}$, i.e., for $K, L \in \mathscr{K}^{n}$,

$$
d(K, L)=|h(K, u)-h(L, u)|_{\infty},
$$

where $|\cdot|_{\infty}$ denotes the sup-norm on the space of continuous functions $C\left(S^{n-1}\right)$.

2.1. Mixed Volumes. If $K_{i}(i=1,2, \ldots, r)$ are compact convex subset and $\lambda_{i}(i=1,2, \ldots, r)$ are nonnegative real numbers, then fundamental importance is the fact that the volume of $\sum_{i=1}^{r} \lambda_{i} K_{i}$ is a homogeneous polynomial in $\lambda_{i}$ given by (see, e.g., [53])

$$
V\left(\lambda_{1} K_{1}+\cdots+\lambda_{n} K_{n}\right)=\sum_{i_{1}, \ldots, i_{n}} \lambda_{i_{1}} \ldots \lambda_{i_{n}} V_{i_{1} \ldots i_{n}}
$$

where the sum is taken over all $n$-tuples $\left(i_{1}, \ldots, i_{n}\right)$ of positive integers not exceeding $r$. The coefficient $V_{i_{1} \ldots i_{n}}$ depends only on the bodies $K_{i_{1}}, \ldots, K_{i_{n}}$ and is uniquely determined by (29); it is called the mixed volume of $K_{i_{1}}, \ldots, K_{i_{n}}$ and is written as $V\left(K_{i_{1}}, \ldots, K_{i_{n}}\right)$. If $K_{1}=\ldots=K_{n-i}=K$ and $K_{n-i+1}=\ldots=$ $K_{n}=L$, then the mixed volume $V\left(K_{1}, \ldots, K_{n}\right)$ is written as $V_{i}(K, L)$. If $K_{1}=\cdots=K_{n-i}=K, K_{n-i+1}=\cdots=K_{n}=$ $B$, the mixed volume $V_{i}(K, B)$ is written as $W_{i}(K)$ and called quermassintegrals (or $i$ th mixed quermassintegrals) of $K$. If $K_{1}=\cdots=K_{n-i-1}=K, K_{n-i}=\cdots=K_{n-1}=B$, and $K_{n}=L$, the mixed volume $V(K, \ldots, K, B, \ldots, B, L)$, with $i$ copies of $B$ and $(n-i-1)$ copies of $K$, is written as $W_{i}(K, L)$ and called as the mixed quermassintegrals.

Aleksandrov [51] (also see Fenchel and Jessen [52], Busemann [54], and Schneider [55]) have shown that, for $K \in \mathscr{K}^{n}$ and $i=0,1, \ldots, n-1$, there exists a regular Borel measure (ith mixed surface area measure) $S_{i}(K, \cdot)$ on $S^{n-1}$, such that the mixed quermassintegrals $W_{i}(K, L)$ have the following representation:

$$
\begin{aligned}
W_{i}(K, L) & =\frac{1}{n-i} \lim _{\varepsilon \rightarrow 0^{+}} \frac{W_{i}(K+\varepsilon L)-W_{i}(K)}{\varepsilon} \\
& =\frac{1}{n} \int_{S^{n-1}} h(L, u) d S_{i}(K, u) .
\end{aligned}
$$


A Minkowski inequality for mixed quermassintegrals states that, for $K, L \in \mathscr{K}^{n}$ and $0 \leq i<n-1$,

$$
W_{i}(K, L)^{n-i} \geq W_{i}(K)^{n-i-1} W_{i}(L),
$$

with equality if and only if $K$ and $L$ are homothetic (see $[54,56])$. A Brunn-Minkowski inequality for mixed quermassintegrals states that, for $K, L \in \mathscr{K}^{n}$ and $0 \leq i<n-1$,

$$
W_{i}(K+L)^{1 /(n-i)} \geq W_{i}(K)^{1 /(n-i)}+W_{i}(L)^{1 /(n-i)},
$$

with equality if and only if $K$ and $L$ are homothetic (see [2]).

2.2. $L_{p}$-Mixed Volumes. The best-known inequality concerning volumes of compact convex sets is the BrunnMinkowski inequality, stating that if $K$ and $L$ are compact convex sets in $\mathbb{R}^{n}$, then

$$
V(K+L)^{1 / n} \geq V(K)^{1 / n}+V(L)^{1 / n},
$$

with equality if and only if $K$ and $L$ are homothetic.

The mixed volume $V_{1}(K, L)$ of compact convex sets $K, L$ is defined by

$$
\begin{aligned}
V_{1}(K, L) & =: \frac{1}{n} \lim _{\varepsilon \rightarrow 0^{+}} \frac{V(K+\varepsilon L)-V(K)}{\varepsilon} \\
& =\frac{1}{n} \int_{S^{n-1}} h(L, u) d S(K, u) .
\end{aligned}
$$

The Minkowski's first inequality for $K, L$ states that

$$
V_{1}(K, L) \geq V(K)^{(n-1) / n} V(L)^{1 / n},
$$

with equality if and only if $K$ and $L$ are homothetic.

From the Brunn-Minkowski inequality, using only a little calculus, one can also derive Minkowski's second inequality (see $[57, \mathrm{p} .370]$ )

$$
V_{1}(K, L)^{2} \geq V(K) V(K, \ldots, K, L, L),
$$

with equality if and only if $K$ and $L$ are homothetic and where $K$ appears $(n-2)$ times.

The middle expression in (34) is the first variation of the volume of $K$ with respect to $L$ and the right-hand side of (34) is its integral representation. The $L_{p}$-Brunn-Minkowski theory received its greatest single impetus when Lutwak [49] found the appropriate $L_{p}$ versions of (34) and (35) and their ingredients. By replacing Minkowski addition and scalar multiplication in (34) by $L_{p}$ addition and its scalar addition and its scalar multiplication,

$$
{ }_{p} \varepsilon \cdot L=\varepsilon^{1 / p} L,
$$

where $p \geq 1$. He established the $L_{p}$ Minkowski mixed volume inequality.

$$
V_{p}(K, L) \geq V(K)^{(n-p) / n} V(L)^{p / n},
$$

with equality if and only if $K$ and $L$ are homothetic, where

$$
V_{p}(K, L):=\frac{p}{n} \lim _{\varepsilon \longrightarrow 0^{+}} \frac{V\left(K+_{p} \varepsilon \cdot L\right)-V(K)}{\varepsilon},
$$

and showed that the $L_{p}$-mixed volume has the following integral representation:

$$
V_{p}(K, L)=\frac{1}{n} \int_{S^{n-1}} h(L, u)^{p} h(K, u)^{1-p} d S(K, u) .
$$

In particular, in the $L_{p}$-Brunn-Minkowski theory, $S(K, \cdot)$ is replaced by the $p$-surface area measure $S_{p}(K, \cdot)$ given by

$$
d S_{p}(K, \cdot)=h(K, u)^{1-p} d S(K ; u) .
$$

Hence, the integral representation (40) becomes

$$
V_{p}(K, L)=\frac{1}{n} \int_{S^{n-1}} h(L, u)^{p} d S_{p}(K, u) .
$$

2.3. The Mixed p-Quermassintegrals. Mixed quermassintegrals are, of course, the first variation of the ordinary quermassintegrals, with respect to Minkowski addition. The mixed quermassintegrals $W_{p, 0}(K, L), W_{p, 1}(K, L)$, $\ldots, W_{p, n-1}(K, L)$ are the first variation of the ordinary quermassintegrals, with respect to Fiery addition: for $K, L \in \mathscr{K}_{o o}^{n}$ and real $p \geq 1$, defined by (see, e.g., [49])

$$
W_{p, i}(K, L)=\frac{p}{n-i} \lim _{\varepsilon 0_{0}^{+}} \frac{W_{i}\left(K+_{p} \varepsilon \cdot L\right)-W_{i}(K)}{\varepsilon} .
$$

For $p \geq 1$ and $0 \leq i<n$ and each $K \in \mathscr{K}_{o o}^{n}$, there exists a regular Borel measure $S_{p, i}(K ; u)$ on $S^{n-1}$, such that the mixed quermassintegral $W_{p, i}(K, L)$ has the following integral representation:

$$
W_{p, i}(K, L)=\frac{1}{n} \int_{S^{n-1}} h(L, u)^{p} d S_{p, i}(K, u),
$$

for all $L \in \mathscr{K}_{o o}^{n}$. The measure $S_{p, i}(K, \cdot)$ is absolutely continuous with respect to $S_{i}(K, \cdot)$ and has Radon-Nikodym derivative

$$
\frac{d S_{p, i}(K, \cdot)}{d S_{i}(K, \cdot)}=h(K, \cdot)^{1-p}
$$

where $S_{i}(K, \cdot)$ is a regular Boel measure on $S^{n-1}$. The measure $S_{n-1}(K, \cdot)$ is independent of the body $K$ and is just ordinary Lebesgue measure, $S$, on $S^{n-1} . S_{i}(B, \cdot)$ denotes the $i$-th surface area measure of the unit ball in $\mathbb{R}^{n}$. In fact, $S_{i}(B, \cdot)=S$ for all $i$. The surface area measure $S_{0}(K, \cdot)$ will frequently be written simply as $S(K, \cdot)$. When $i=0, S_{p, i}(K, \cdot)$ is just the $p$-surface area measure $S_{p}(K, \cdot)$ (see $\left.[15,16]\right)$. Obviously, putting $i=0$ in (44), the mixed $p$-quermassintegrals $W_{p, i}(K, L)$ become the $L_{p}$-mixed volume $V_{p}(K, L)$.

A fundamental inequality for mixed $p$-quermassintegrals states that (see [49]), for $K, L \in \mathscr{K}_{o o}^{n}, p \geq 1$ and $0 \leq i<n$,

$$
W_{p, i}(K, L)^{n-i} \geq W_{i}(K)^{n-i-p} W_{i}(L)^{p},
$$

with equality if and only if $K$ and $L$ are homothetic. The $L_{p^{-}}$ Brunn-Minkowski inequality for quermassintegrals was also established. If $K, L \in \mathscr{K}_{o o}^{n}$ and $p \geq 1$ and $0 \leq i<n$, then

$$
W_{i}\left(K+{ }_{p} L\right)^{p /(n-i)} \geq W_{i}(K)^{p /(n-i)}+W_{i}(L)^{p /(n-i)},
$$

with equality if and only if $K$ and $L$ are homothetic. 


\section{Orlicz Linear Combination}

Throughout the paper, let $\Phi_{m}, m \in \mathbb{N}$, denote the set of convex functions $\varphi:[0, \infty)^{m} \longrightarrow[0, \infty)$ that are strictly increasing in each variable and satisfy $\varphi(0, \ldots, 0)=0$ and $\varphi(0, \ldots, 1, \ldots, 0)=1$. When $m=1$, we shall write $\Phi$ instead of $\Phi_{1}$.

Let $m \geq 2, \varphi \in \Phi_{m}, K_{j} \in \mathscr{K}_{o}^{n}$, and $j=1, \ldots, m$; the Orlicz addition of $K_{1}, \ldots, K_{m}$, denoted by $+_{\varphi}\left(K_{1}, \ldots, K_{m}\right)$, is defined by ([25])

$$
\begin{aligned}
& h\left(+_{\varphi}\left(K_{1}, \ldots, K_{m}\right), x\right) \\
& =\inf \left\{\lambda>0: \varphi\left(\frac{h\left(K_{1}, x\right)}{\lambda}, \ldots, \frac{h\left(K_{m}, x\right)}{\lambda}\right) \leq 1\right\},
\end{aligned}
$$

for $x \in \mathbb{R}^{n}$.

Equivalently, the Orlicz radial addition $+_{\varphi}\left(K_{1}, \ldots, K_{m}\right)$ can be defined implicitly (and uniquely) by

$$
\begin{gathered}
\varphi\left(\frac{h\left(K_{1}, x\right)}{h\left(+_{\varphi}\left(K_{1}, \ldots, K_{m}\right), x\right)}, \ldots,\right. \\
\left.\frac{h\left(K_{m}, x\right)}{h\left(+_{\varphi}\left(K_{1}, \ldots, K_{m}\right), x\right)}\right)=1,
\end{gathered}
$$

if $h\left(K_{1}, x\right)+\cdots+h\left(K_{m}, x\right)>0$ and by $h\left(+{ }_{\varphi}\left(K_{1}, \ldots, K_{m}\right), x\right)=$ 0 , if $h\left(K_{1}, x\right)=\cdots=h\left(K_{m}, x\right)=0$, for all $x \in \mathbb{R}^{n}$.

An important special case is obtained when

$$
\varphi\left(x_{1}, \ldots, x_{m}\right)=\sum_{j=1}^{m} \varphi\left(x_{j}\right),
$$

for some fixed $\varphi \in \Phi$ such that $\varphi(1)=1$, and in this case write $+_{\varphi}\left(K_{1}, \ldots, K_{m}\right)=K_{1}+_{\varphi} \cdots+_{\varphi} K_{m}$. This means that $K_{1}+{ }_{\varphi} \cdots+{ }_{\varphi} K_{m}$ is defined either by

$$
\begin{aligned}
& h\left(K_{1}+{ }_{\varphi} \cdots+{ }_{\varphi} K_{m}, x\right) \\
& \quad=\inf \left\{\lambda>0: \sum_{j=1}^{m} \varphi\left(\frac{h\left(K_{j}, x\right)}{\lambda}\right) \leq 1\right\},
\end{aligned}
$$

for all $x \in \mathbb{R}^{n}$, or by the corresponding special case of (49). From (51), it follows easy that

$$
\sum_{j=1}^{m} \varphi\left(\frac{h\left(K_{j}, x\right)}{\lambda}\right)=1,
$$

if and only if

$$
\lambda=h\left(K_{1}+{ }_{\varphi} \cdots+{ }_{\varphi} K_{m}, x\right) .
$$

The Orlicz addition $+_{\varphi}$ is continuous, monotonic, $G L(n)$ covariant, and projection covariant. The Orlicz linear combination was defined by (see [25])

$$
\begin{aligned}
& h\left(+{ }_{\varphi}\left(K_{1}, \ldots, K_{m}, \alpha_{1}, \ldots, \alpha_{m}\right), x\right) \\
& \quad=\inf \left\{\lambda>0: \sum_{j=1}^{m} \alpha_{j} \varphi\left(\frac{h\left(K_{j}, x\right)}{\lambda}\right) \leq 1\right\},
\end{aligned}
$$

for all $x \in \mathbb{R}^{n}$, where $K_{1}, \ldots, K_{m} \in \mathscr{K}_{o}^{n}, \alpha_{j} \geq 0$, and $\varphi \in \Phi$. Definition (54) corresponds to taking the function $\varphi$ in (48) and (49) to be

$$
\varphi\left(x_{1}, \ldots, x_{m}\right)=\sum_{j=1}^{m} \alpha_{j} \varphi\left(x_{j}\right),
$$

where $\varphi \in \Phi_{m}$.

\section{Orlicz Multiple Mixed Volumes}

Let us introduce Orlicz multiple mixed volume.

Definition 1. For $\varphi \in \Phi$, we define Orlicz multiple mixed volume, denoted by $V_{\varphi}\left(K_{1}, \ldots, K_{n}, L_{n}\right)$, defined by

$$
\begin{aligned}
& V_{\varphi}\left(K_{1}, \ldots, K_{n}, L_{n}\right)=: \frac{1}{n} \\
& \cdot \int_{S^{n-1}} \varphi\left(\frac{h\left(K_{n}, u\right)}{h\left(L_{n}, u\right)}\right) h\left(L_{n}, u\right) \\
& \cdot d S\left(K_{1}, \ldots, K_{n-1} ; u\right),
\end{aligned}
$$

for all $K_{1}, \ldots, K_{n} \in \mathscr{K}_{o}^{n}$ and $L_{n} \in \mathscr{K}_{o o}^{n}$.

To derive this Definition 1, we need the following lemmas.

Lemma 2 ([25]). If $K, L \in \mathscr{K}_{o}^{n}$, then

$$
K+{ }_{\varphi, \varepsilon} L \longrightarrow K
$$

in the Hausdorff metric as $\varepsilon \longrightarrow 0^{+}$.

Lemma 3. If $K_{1}, \ldots, K_{n} \in \mathscr{K}_{o}^{n}, L_{n} \in \mathscr{K}_{o o}^{n}$, and $\varphi \in \Phi$, then for $\varepsilon>0$

$$
\begin{aligned}
& \left.\varphi_{l}^{\prime}(1) \cdot \frac{d}{d \varepsilon}\right|_{\varepsilon=0^{+}} V\left(K_{1}, \ldots, K_{n-1}, L_{n}+_{\varphi, \varepsilon} K_{n}\right)=\frac{1}{n} \\
& \cdot \int_{S^{n-1}} \varphi\left(\frac{h\left(K_{n}, u\right)}{h\left(L_{n}, u\right)}\right) h\left(L_{n}, u\right) \\
& \cdot d S\left(K_{1}, \ldots, K_{n-1} ; u\right) .
\end{aligned}
$$

Proof. Suppose $\varepsilon>0, K_{n} \in \mathscr{K}_{o}^{n}, L_{n} \in \mathscr{K}_{o o}^{n}$, and $u \in S^{n-1}$; let

$$
h_{\varepsilon}=h\left(L_{n}{ }_{\varphi, \varepsilon} K_{n}, u\right) \text {. }
$$

Since

$$
\frac{h\left(L_{n}, u\right)}{h_{\varepsilon}}=\varphi^{-1}\left(1-\varepsilon \varphi\left(\frac{h\left(K_{n}, u\right)}{h_{\varepsilon}}\right)\right)
$$

and from Lemma 2 and noting that $\varphi$ is continuous function, we obtain

$$
\begin{aligned}
& \left.\frac{d}{d \varepsilon}\right|_{\varepsilon=0^{+}} V\left(K_{1}, \ldots, K_{n-1}, L_{n}{ }_{\varphi, \varepsilon} K_{n}\right)=\frac{1}{n} \lim _{\varepsilon \longrightarrow 0^{+}} \int_{S^{n-1}} \frac{h_{\varepsilon}}{\varepsilon} \\
& . \frac{h_{\varepsilon}-h\left(L_{n}, u\right)}{h_{\varepsilon}} d S\left(K_{1}, \ldots, K_{n-1} ; u\right)=\frac{1}{n} \\
& \quad \cdot \lim _{\varepsilon \longrightarrow 0^{+}} \int_{S^{n-1}} h_{\varepsilon} \cdot \varphi\left(\frac{h\left(K_{n}, u\right)}{h_{\varepsilon}}\right)
\end{aligned}
$$




$$
\begin{aligned}
& \cdot \frac{1-\varphi^{-1}\left(1-\varepsilon \varphi\left(h\left(K_{n}, u\right) / h_{\varepsilon}\right)\right)}{1-\left(1-\varepsilon \varphi\left(h\left(K_{n}, u\right) / h_{\varepsilon}\right)\right)} \\
& \cdot d S\left(K_{1}, \ldots, K_{n-1} ; u\right)=\frac{1}{n} \int_{S^{n-1}} h\left(L_{n}, u\right) \\
& \cdot \varphi\left(\frac{h\left(K_{n}, u\right)}{h\left(L_{n}, u\right)}\right) \lim _{y \rightarrow 1^{-}} \frac{1-y}{\varphi(1)-\varphi(y)} d S\left(K_{1}, \ldots,\right. \\
& \left.K_{n-1} ; u\right)=\frac{1}{n \varphi_{l}^{\prime}(1)} \int_{S^{n-1}} \varphi\left(\frac{h\left(K_{n}, u\right)}{h\left(L_{n}, u\right)}\right) h\left(L_{n}, u\right) \\
& \cdot d S\left(K_{1}, \ldots, K_{n-1} ; u\right),
\end{aligned}
$$

where

$$
y=\varphi^{-1}\left(1-\varepsilon \varphi\left(\frac{h\left(K_{n}, u\right)}{h_{\varepsilon}}\right)\right) .
$$

For any $K_{1}, \ldots, K_{n} \in \mathscr{K}_{o}^{n}, L_{n} \in \mathscr{K}_{o o}^{n}$, and $\varphi \in \Phi$, we see that either side of (61) is equal to $V_{\varphi}\left(K_{1}, \ldots, K_{n}, L_{n}\right)$, and so this new Orlicz multiple dual mixed volume $V_{\varphi}\left(K_{1}, \ldots, K_{n}, L_{n}\right)$ has been born. Hence, the equation in Definition 1 follows immediately.

This theorem plays a central role in our deriving the Orlicz multiple mixed volume. Hence, we give the second kind of different proof.

Second Proof. One has

$$
\frac{d h_{\varepsilon}}{d \varepsilon}=\frac{d}{d \varepsilon}\left(\frac{h\left(L_{n}, u\right)}{\varphi^{-1}\left(1-\varepsilon \varphi\left(\frac{h\left(K_{n}, u\right)}{h_{\varepsilon}}\right)\right)}\right)=\frac{h\left(L_{n}, u\right) \frac{d \varphi^{-1}(y)}{d y}\left[\varphi\left(\frac{h\left(K_{n}, u\right)}{h_{\varepsilon}}\right)-\varepsilon \cdot \frac{d \varphi(z)}{d z} \frac{h\left(K_{n}, u\right)}{h_{\varepsilon}^{2}} \frac{d h_{\varepsilon}}{d \varepsilon}\right]}{\left(\varphi^{-1}\left(1-\varepsilon \varphi\left(\frac{h\left(K_{n}, u\right)}{h_{\varepsilon}}\right)\right)\right)^{2}} .
$$

where

and

$$
z=\frac{h\left(K_{n}, u\right)}{h_{\varepsilon}} .
$$

$$
y=1-\varepsilon \varphi\left(\frac{h\left(K_{n}, u\right)}{h_{\varepsilon}}\right),
$$

Hence

$$
\frac{d h_{\varepsilon}}{d \varepsilon}=\frac{h\left(L_{n}, u\right) \frac{d \varphi^{-1}(y)}{d y} \varphi\left(\frac{h\left(K_{n}, u\right)}{h_{\varepsilon}}\right)}{\left(\varphi^{-1}\left(1-\varepsilon \varphi\left(\frac{h\left(K_{n}, u\right)}{h_{\varepsilon}}\right)\right)\right)^{2}+\varepsilon \cdot \frac{h\left(K_{n}, u\right) h\left(L_{n}, u\right)}{h_{\varepsilon}^{2}} \frac{d \varphi^{-1}(y)}{d y} \frac{d \varphi(z)}{d z}} .
$$

Therefore

$$
\begin{aligned}
& \left.\frac{d}{d \varepsilon}\right|_{\varepsilon=0^{+}} V\left(K_{1}, \ldots, K_{n-1}, L_{n}+{ }_{\varphi, \varepsilon} K_{n}\right) \\
& \quad=\frac{1}{n} \lim _{\varepsilon \rightarrow 0^{+}} \int_{S^{n-1}} \frac{h_{\varepsilon}-h\left(L_{n}, u\right)}{\varepsilon} d S\left(K_{1}, \ldots, K_{n-1} ; u\right) \\
& \quad=\frac{1}{n_{\varepsilon} \longrightarrow 0^{+}} \int_{S^{n-1}} \frac{d h_{\varepsilon}}{d \varepsilon} d S\left(K_{1}, \ldots, K_{n-1} ; u\right) .
\end{aligned}
$$

On the other hand, noting that $y \longrightarrow 1^{-}$as $\varepsilon \longrightarrow 0^{+}$, we have

$$
\frac{d \varphi^{-1}(y)}{d \varepsilon}=\lim _{y \rightarrow 1^{-}} \frac{\varphi^{-1}(y)-\varphi^{-1}(1)}{y-1}=\frac{1}{\varphi_{l}^{\prime}(1)} .
$$

From (64), (66), (67), (68), and Lemma 2 and noticing that $\varphi$ and $\varphi^{-1}$ are continuous functions, (58) yields easy.
Lemma 4. If $K_{1}, \ldots, K_{n} \in \mathscr{K}_{o}^{n}, L_{n} \in \mathscr{K}_{o o}^{n}$, and $\varphi \in \Phi$, then

$$
\begin{aligned}
& V_{\varphi}\left(K_{1}, \ldots, K_{n}, L_{n}\right) \\
& \quad=\left.\varphi_{l}^{\prime}(1) \cdot \frac{d}{d \varepsilon}\right|_{\varepsilon=0^{+}} V\left(K_{1}, \ldots, K_{n-1}, L_{n}{ }_{\varphi, \varepsilon} K_{n}\right) .
\end{aligned}
$$

Proof. This follows immediately from Definition 1 and Lemma 3.

Lemma 5 ([25]). If $K_{i}, L_{i} \in \mathscr{K}_{o}^{n}$ and $K_{i} \longrightarrow K, L_{i} \longrightarrow L$ as $i \longrightarrow \infty$, then

$$
K_{i}+_{\varphi, \varepsilon} L i K{ }_{\varphi, \varepsilon} L, \quad \text { as } i \longrightarrow \infty,
$$

for $\varepsilon>0$.

Let us list some important properties of the Orlicz multiple mixed volume. 
Lemma 6. If $K_{1}, \ldots, K_{n}, L_{n}, K, L \in \mathscr{K}_{o}^{n}, \lambda_{1}, \ldots, \lambda_{n} \geq 0$, and $\varphi \in \Phi$, then one has the following:

(1) $V_{\varphi}\left(K_{1}, \ldots, K_{n}, L_{n}\right) \geq 0$.

(2) $V_{\varphi}(K, K, \ldots, K)=V(K)$.

(3) for $K \subset L$

$$
V_{\varphi}\left(K, K_{2} \cdots, K_{n}, L_{n}\right) \leq V_{\varphi}\left(L, K_{2} \cdots, K_{n}, L_{n}\right) .
$$

(4)

$$
\begin{aligned}
V_{\varphi} & \left(\lambda_{1} K_{1}, \ldots, \lambda_{n} K_{n}, \lambda_{n} L_{n}\right) \\
& =\lambda_{1} \cdots \lambda_{n} V_{\varphi}\left(K_{1}, \ldots, K_{n}, L_{n}\right) .
\end{aligned}
$$

(5) The Orlicz multiple mixed volume $V_{\varphi}\left(K_{1}, \ldots, K_{n}, L_{n}\right)$ is continuous function of $K_{1}, \ldots, K_{n}, L_{n}$.

(6)

$$
\begin{aligned}
V_{\varphi} & \left(\lambda_{1} K+\lambda_{2} L, K_{2} \cdots, K_{n}, L_{n}\right) \\
= & \lambda_{1} V_{\varphi}\left(K, K_{2}, \ldots, K_{n}, L_{n}\right) \\
& +\lambda_{2} V_{\varphi}\left(L, K_{2}, \ldots, K_{n}, L_{n}\right) .
\end{aligned}
$$

This shows that the Orlicz multiple mixed volume $V_{\varphi}\left(K_{1}, \ldots, K_{n}, L_{n}\right)$ is linear in its $(n-1)$ variables in front.

Proof. From Definition 1, it immediately gives (1), (2), and (3). Combining Definition 1 with the fact (see [58])

$$
\begin{aligned}
& S\left(\lambda_{1} K_{1}, \ldots, \lambda_{n-1} K_{n-1}, \cdot\right) \\
& \quad=\lambda_{1} \cdots \lambda_{n-1} S\left(K_{1}, \ldots, K_{n-1}, \cdot\right),
\end{aligned}
$$

it yields (4) directly.
Suppose $L_{\text {in }} \longrightarrow L_{n}, K_{i j} \longrightarrow K_{j}$ as $i \longrightarrow \infty$ where $j=$ $1, \ldots, n$; combining Definition 1 and Lemma 5 with the facts

$$
\begin{gathered}
V\left(K_{i 1}, \ldots, K_{i n}, K_{i n}{ }_{\varphi, \varepsilon} L_{i n},\right) \longrightarrow \\
V\left(K_{1}, \ldots, K_{n}, K_{n}{ }_{\varphi, \varepsilon} L_{n},\right)
\end{gathered}
$$

and

$$
V\left(K_{i 1}, \ldots, K_{i n}, L_{i n},\right) \longrightarrow V\left(K_{1}, \ldots, K_{n}, L_{n}\right)
$$

as $i \longrightarrow \infty$, it yields (5) directly.

Combining Definition 1 and noting that the mixed area measure is Minkowski linear in each of its arguments (see [59])

$$
\begin{aligned}
& S\left(K+L, K_{2} \cdots, K_{n-1}, \cdot\right) \\
& \quad=S\left(K, K_{2}, \ldots, K_{n-1}, \cdot\right)+S\left(L, K_{2} \cdots, K_{n-1}, \cdot\right)
\end{aligned}
$$

it yields (6) directly.

Lemma 7 ([25]). Suppose $K, L \in \mathscr{K}_{o}^{n}$ and $\varepsilon>0$. If $\varphi \in \Phi$, then for $A \in G L(n)$

$$
A\left(K+_{\varphi, \varepsilon} L\right)=A K+_{\varphi, \varepsilon} A L .
$$

We easy find that Orlicz multiple dual mixed volume $V_{\varphi}\left(K_{1}, K_{2}, \cdots, K_{n}, L_{n}\right)$ is invariant under simultaneous unimodular centro-affine transformation.

Lemma 8. If $K_{1}, \ldots, K_{n}, L_{n} \in \mathscr{K}_{o}^{n}$ and $\varphi \in \Phi$, then, for $A \in$ $S L(n)$,

$$
V_{\varphi}\left(A K_{1}, \ldots, A K_{n}, A L_{n}\right)=V_{\varphi}\left(K_{1}, \ldots, K_{n}, L_{n}\right) .
$$

Proof. From (69) and Lemma 7, we have, for $A \in S L(n)$,

$$
\begin{aligned}
V_{\varphi}\left(A K_{1}, \ldots, A K_{n}, A L_{n}\right) & =\varphi_{l}^{\prime}(1) \lim _{\varepsilon \longrightarrow 0^{+}} \frac{V\left(A K_{1}, \ldots, A K_{n-1}, A L_{n}+_{\varphi, \varepsilon} A K_{n}\right)-V\left(A K_{1}, \ldots, A K_{n-1}, A L_{n}\right)}{\varepsilon} \\
& =\varphi_{l}^{\prime}(1) \lim _{\varepsilon \longrightarrow 0^{+}} \frac{V\left(A K_{1}, \ldots, A K_{n-1}, A\left(L_{n}+{ }_{\varphi, \varepsilon} K_{n}\right)\right)-V\left(K_{1}, \ldots, K_{n-1}, L_{n}\right)}{\varepsilon} \\
& =\varphi_{l}^{\prime}(1) \lim _{\varepsilon \longrightarrow 0^{+}} \frac{V\left(K_{1}, \ldots, K_{n-1}, L_{n}{ }^{+}{ }_{\varphi, \varepsilon} K_{n}\right)-V\left(K_{1}, \ldots, K_{n-1}, L_{n}\right)}{\varepsilon}=V_{\varphi}\left(K_{1}, \ldots, K_{n}, L_{n}\right) .
\end{aligned}
$$

$$
\begin{aligned}
\lim _{\varepsilon \longrightarrow 0^{+}} & \frac{V_{1}\left(K, K+_{\varphi, \varepsilon} L\right)-V(K)}{\varepsilon} \\
& =\frac{1}{n} \lim _{\varepsilon \longrightarrow 0^{+}} \frac{V\left(K+_{\varphi, \varepsilon} L\right)-V(K)}{\varepsilon} .
\end{aligned}
$$

$$
V_{\varphi}(K, L)=\frac{\varphi_{l}^{\prime}(1)}{n} \lim _{\varepsilon \longrightarrow 0^{+}} \frac{V\left(K{ }_{\varphi, \varepsilon} L\right)-V(K)}{\varepsilon} .
$$

Lemma 10. If $K \in \mathscr{K}_{o o}^{n}, L \in \mathscr{K}_{o}^{n}$, and $\varphi \in \Phi$, then
Proof. Suppose $\varepsilon>0, K \in \mathscr{K}_{o o}^{n}, L \in \mathscr{K}_{o}^{n}$, and $u \in S^{n-1}$; let

$$
\bar{h}_{\varepsilon}=h\left(K+_{\varphi, \varepsilon} L, u\right) \text {. }
$$


From (5), (35), (66), (68), and Lemma 9, we obtain

$$
\begin{aligned}
\lim _{\varepsilon \longrightarrow 0^{+}} & \frac{V_{1}\left(K, K+_{\varphi, \varepsilon} L\right)-V(K)}{\varepsilon} \\
= & \frac{1}{n} \int_{S^{n-1}} \lim _{\varepsilon \longrightarrow 0^{+}} \frac{\bar{h}_{\varepsilon}-h(K, u)}{\varepsilon} d S(K ; u) \\
= & \frac{1}{n} \int_{S^{n-1}} \lim _{\varepsilon \longrightarrow 0^{+}} \frac{d \bar{h}_{\varepsilon}}{d \varepsilon} d S(K ; u) \\
= & \frac{1}{n \varphi_{l}^{\prime}(1)} \int_{S^{n-1}} \varphi\left(\frac{h(L, u)}{h(K, u)}\right) h(K, u) d S(K ; u) \\
= & \frac{1}{n} \lim _{\varepsilon \longrightarrow 0^{+}} \frac{V\left(K+{ }_{\varphi, \varepsilon} L\right)-V(K)}{\varepsilon} .
\end{aligned}
$$

Lemma 11. Let $K_{1}, \ldots, K_{n} \in \mathscr{K}_{o}^{n}$ and $L_{n} \in \mathscr{K}_{\text {oo }}^{n}$. If $K_{1}=\cdots=$ $K_{n-1}=K, K_{n}=L$, and $L_{n}=K$, then

$$
V_{\varphi}\left(K_{1}, \cdots, K_{n}, L_{n}\right)=V_{\varphi}(K, L) .
$$

Proof. On the one hand, putting $K_{1}=\cdots=K_{n-1}=K, K_{n}=$ $L$, and $L_{n}=K$ in (69) and noting Lemmas 10 and 9, it follows that

$$
\begin{aligned}
V_{\varphi} & \left(K_{1}, \ldots, K_{n}, L_{n}\right) \\
& =\varphi_{l}^{\prime}(1) \lim _{\varepsilon \longrightarrow 0^{+}} \frac{V_{1}\left(K, K+_{\varphi, \varepsilon} L\right)-V(K)}{\varepsilon} \\
& =\frac{\varphi_{l}^{\prime}(1)}{n} \lim _{\varepsilon \longrightarrow 0^{+}} \frac{V\left(K+_{\varphi, \varepsilon} L\right)-V(K)}{\varepsilon}=V_{\varphi}(K, L) .
\end{aligned}
$$

On the other hand, putting $K_{1}=\cdots=K_{n-1}=K, K_{n}=L$, and $L_{n}=K$ in Definition 1 and in view of (5), then

$$
\begin{aligned}
& V_{\varphi}\left(K_{1}, \ldots, K_{n}, L_{n}\right) \\
& \quad=\frac{1}{n} \int_{S^{n-1}} \varphi\left(\frac{h(L, u)}{h(K, u)}\right) h(K, u) d S\left(K_{1}, \ldots, K_{n-1} ; u\right) \\
& =V_{\varphi}(K, L) .
\end{aligned}
$$

Combining (86), (87), and Lemma 3, this shows that

$$
V_{\varphi}\left(K_{1}, \cdots, K_{n}, L_{n}\right)=V_{\varphi}(K, L)
$$

if $K_{1}=\cdots=K_{n-1}=K, K_{n}=L$, and $L_{n}=L$.

Lemma 12. If $K \in \mathscr{K}_{o o}^{n}, L \in \mathscr{K}_{o}^{n}$ and $\varphi \in \Phi$, and $0 \leq i<n$, then

$$
\begin{aligned}
& V_{\varphi}(K[n-i-1], B[i], L, K) \\
& \quad=\frac{1}{n} \int_{S^{n-1}} \varphi\left(\frac{h(L, u)}{h(K, u)}\right) h(K, u) d S_{i}(K ; u)
\end{aligned}
$$

Proof. On the one hand, putting $K_{1}=\cdots=K_{n-i-1}=$ $K, K_{n-i}=\cdots=K_{n-1}=B, L_{n}=K$, and $K_{n}=L$ in (69), from (31), (66), and (68), we obtain for $\varphi \in \Phi$

$$
\begin{aligned}
& V_{\varphi}(K[n-i-1], B[i], L, K) \\
& =\varphi_{l}^{\prime}(1) \lim _{\varepsilon \longrightarrow 0^{+}} \frac{W_{i}\left(K, K+_{\varphi, \varepsilon} L\right)-W_{i}(K)}{\varepsilon} \\
& =\frac{1}{n} \varphi_{l}^{\prime}(1) \int_{S^{n-1}} \lim _{\varepsilon \longrightarrow 0^{+}} \frac{h\left(K+_{\varphi, \varepsilon} L\right)-h(K, u)}{\varepsilon} d S_{i}(K ; u) \\
& =\frac{1}{n} \varphi_{l}^{\prime}(1) \int_{S^{n-1}} \lim _{\varepsilon \longrightarrow 0^{+}} \frac{d \bar{h}_{\varepsilon}}{\varepsilon} d S_{i}(K ; u) \\
& =\frac{1}{n} \int_{S^{n-1}} \varphi\left(\frac{h(L, u)}{h(K, u)}\right) h(K, u) d S_{i}(K ; u) .
\end{aligned}
$$

On the other hand, putting $K_{1}=\cdots=K_{n-i-1}=K, K_{n-i}=$ $\cdots=K_{n-1}=B, L_{n}=K$, and $K_{n}=L$, from Definition 1, we have for $\varphi \in \Phi$

$$
\begin{aligned}
V_{\varphi} & (K[n-i-1], B[i], L, K) \\
= & \frac{1}{n} \int_{S^{n-1}} \varphi\left(\frac{h(L, u)}{h(K, u)}\right) h(K, u) d S_{i}(K ; u),
\end{aligned}
$$

where $K \in \mathscr{K}_{o o}^{n}, L \in \mathscr{K}_{o}^{n}$ and $\varphi \in \Phi$, and $0 \leq i<n$.

Here, we denote the Orlicz multiple mixed volume $V_{\varphi}(K[n-i-1], B[i], L, K)$ by $W_{\varphi, i}(K, L)$ and call $W_{\varphi, i}(K, L)$ as Orlicz mixed quermassintegral of convex bodies $K$ and $L$. When $i=0$, Orlicz mixed quermassintegral $W_{\varphi, i}(K, L)$ becomes Orlicz dual mixed volume $V_{\varphi}(K, L)$.

Remark 13. When $\varphi(t)=t^{p}, p=1$, from the integral representation for Orlicz multiple mixed volume $V_{\varphi}\left(K_{1}, \ldots\right.$, $\left.K_{n}, L_{n}\right)$ (see Definition 1$)$, it follows easy that $V_{\varphi}\left(K_{1}, \cdots\right.$, $\left.K_{n}, L_{n}\right)=V\left(K_{1}, \ldots, K_{n}\right)$. On the other hand, when $\varphi(t)=t^{p}$, $p=1$, from (69) and noting that $\varphi_{l}^{\prime}(1)=1$ and $K_{n}+{ }_{\varphi, \varepsilon} L_{n}=$ $K_{n}+\varepsilon \cdot K_{n}$, hence

$$
\begin{aligned}
& V\left(K_{1}, \ldots, K_{n}\right) \\
& =\lim _{\varepsilon \rightarrow 0^{+}} \frac{V\left(K_{1}, \ldots, K_{n-1}, K_{n}+\varepsilon \cdot K_{n}\right)-V\left(K_{1}, \ldots, K_{n}\right)}{\varepsilon} .
\end{aligned}
$$

This is very interesting that the mixed volume is such a limiting form.

On the other hand, taking $L_{n}=K_{n}$ in Definition 1 and noting $\varphi(1)=1, V_{\varphi}\left(K_{1}, \ldots, K_{n}, L_{n}\right)$ also becomes $V\left(K_{1}, \ldots, K_{n}\right)$.

Remark 14. When $\varphi(t)=t^{p}$ and $p \geq 1$, we write $V_{\varphi}\left(K_{1}, \ldots\right.$, $\left.K_{n}, L_{n}\right)$ as $V_{p}\left(K_{1}, \ldots, K_{n}, L_{n}\right)$ and call $V_{p}\left(K_{1}, \ldots, K_{n}, L_{n}\right)$ as $L_{p}$-multiple mixed volume of convex bodies $K_{1}, \ldots, K_{n}, L_{n}$. 
Putting $\varphi(t)=t^{p}, p \geq 1$ in Definition 1 , then

$$
\begin{aligned}
& V_{p}\left(K_{1}, \ldots, K_{n}, L_{n}\right)=\frac{1}{n} \\
& \quad \cdot \int_{S^{n-1}} h\left(L_{n}, u\right)^{1-p} h\left(K_{n}, u\right)^{p} d S\left(K_{1}, \ldots, K_{n-1} ; u\right) .
\end{aligned}
$$

On the other hand, when $\varphi(t)=t^{p}$ and $p \geq 1$, from (69), we get the following a limit of representation of $L_{p}$-multiple mixed volume:

$$
\begin{aligned}
& V_{p}\left(K_{1}, \ldots, K_{n}, L_{n}\right) \\
& =p \lim _{\varepsilon \longrightarrow 0^{+}} \frac{V\left(K_{1}, \ldots, K_{n-1}, L_{n}+{ }_{p} \varepsilon \cdot K_{n},\right)-V\left(K_{1}, \ldots, K_{n-1}, L_{n}\right)}{\varepsilon} .
\end{aligned}
$$

where $p \geq 1$ and $K_{1}, \ldots, K_{n} \in \mathscr{K}_{o}^{n}, L_{n} \in \mathscr{K}_{o o}^{n}$.

Putting $K_{1}=\cdots=K_{n-i-1}=K, K_{n-i}=\cdots=K_{n-1}=B$, $K_{n}=L$, and $L_{n}=K$ in (93) and in view of (45) and (46), we obtain

$$
\begin{aligned}
V_{p} & \left(K_{1}, \ldots, K_{n}, L_{n}\right) \\
& =\frac{1}{n} \int_{S^{n-1}} h(L, u)^{p} h(K, u)^{1-p} d S_{i}(K, u) \\
& =\frac{1}{n} \int_{S^{n-1}} h(L, u)^{p} d S_{p, i}(K, u)=W_{p, i}(K, L) .
\end{aligned}
$$

Obviously, when $L_{n}=K_{n}$ or $p=1$, from (93), $V_{p}\left(K_{1}, \ldots\right.$, $\left.K_{n}, L_{n}\right)$ becomes the mixed volume $V\left(K_{1}, \ldots, K_{n}\right)$. When $K_{1}=\cdots=K_{n-1}=K, K_{n}=L$, and $L_{n}=K, V_{p}\left(K_{1}, \ldots\right.$, $\left.K_{n}, L_{n}\right)$ becomes $V_{p}(K, L)$.

Lemma 15 (Jensen's inequality). Let $\mu$ be a probability measure on a space $X$ and $g: X \longrightarrow I \subset \mathbb{R}$ is a $\mu$-integrable function, where $I$ is a possibly infinite interval. If $\psi: I \longrightarrow \mathbb{R}$ is a convex function, then

$$
\int_{X} \psi(g(x)) d \mu(x) \geq \psi\left(\int_{X} g(x) d \mu(x)\right) .
$$

If $\psi$ is strictly convex, equality holds if and only if $g(x)$ is constant for $\mu$-almost all $x \in X$ (see [60, p.165]).

\section{Orlicz-Aleksandrov-Fenchel Inequality for Orlicz Multiple Mixed Volumes}

Lemma 16. If $K_{1}, \ldots, K_{n} \in \mathscr{K}_{o}^{n}, L_{n} \in \mathscr{K}_{o o}^{n}$, and $\varphi \in \Phi$, then

$$
\frac{V_{\varphi}\left(K_{1}, \ldots, K_{n}, L_{n}\right)}{V\left(K_{1}, \ldots, K_{n-1}, L_{n}\right)} \geq \varphi\left(\frac{V\left(K_{1}, \ldots, K_{n}\right)}{V\left(K_{1}, \ldots, K_{n-1}, L_{n}\right)}\right) \text {. }
$$

If $\varphi$ is strictly convex, equality holds if and only if $K_{n}$ and $L_{n}$ are homothetic.

Proof. For $K_{1}, \ldots, K_{n} \in \mathscr{K}_{o}^{n}$ and any $u \in S^{n-1}$, since

$$
\begin{aligned}
& \frac{1}{n V\left(K_{1}, \ldots, K_{n}\right)} \int_{S^{n-1}} h\left(K_{n}, u\right) d S\left(K_{1}, \ldots, K_{n-1} ; u\right) \\
& \quad=1
\end{aligned}
$$

so $\left(h\left(K_{n}, u\right) / n V\left(K_{1}, \ldots, K_{n}\right)\right) S\left(K_{1}, \ldots, K_{n-1} ; u\right)$ is a probability measure on $S^{n-1}$.

From (7) and (96) and in view of Definition 1, we obtain

$$
\begin{aligned}
& \frac{V_{\varphi}\left(K_{1}, \ldots, K_{n}, L_{n}\right)}{V\left(K_{1}, \ldots, K_{n-1}, L_{n}\right)}=\frac{1}{n V\left(K_{1}, \ldots, K_{n-1}, L_{n}\right)} \\
& \cdot \int_{S^{n-1}} \varphi\left(\frac{h\left(K_{n}, u\right)}{h\left(L_{n}, u\right)}\right) h\left(L_{n}, u\right) d S\left(K_{1}, \ldots, K_{n-1} ; u\right) \\
& \geq \varphi\left(\frac{1}{n V\left(K_{1}, \ldots, K_{n-1}, L_{n}\right)} \int_{S^{n-1}} h\left(K_{n}, u\right)\right. \\
& \left.\cdot d S\left(K_{1}, \ldots, K_{n-1} ; u\right)\right)=\varphi\left(\frac{V\left(K_{1}, \ldots, K_{n}\right)}{V\left(K_{1}, \ldots, K_{n-1}, L_{n}\right)}\right) .
\end{aligned}
$$

Next, we discuss the equality condition of (97). Suppose the equality hold in (97), form the equality condition of Jensen's inequality, it follows that if $\varphi$ is strictly convex the equality in (97) holds if and only if $h\left(K_{n}, u\right) / h\left(L_{n}, u\right)$ is constant for $S(K, \ldots, K ; \cdot)$-almost all $u \in S^{n-1}$. This follows that if $\varphi$ is strictly convex, the equality in (97) holds if and only if $h\left(K_{n}, u\right)$ and $h\left(L_{n}, u\right)$ proportional for $S(K, \ldots, K ; \cdot)$ almost all $u \in S^{n-1}$. This yields that if $\varphi$ is strictly convex, the equality in (97) holds if and only if $K_{n}$ and $L_{n}$ are homothetic.

Conversely, if $\varphi$ is strictly convex, then there exist $r>0$ such that

$$
\frac{h\left(K_{n}, u\right)}{h\left(L_{n}, u\right)}=r
$$

for $S(K, \ldots, K ; \cdot)$-almost all $u \in S^{n-1}$; this shows that

$$
r=\frac{V\left(K_{1}, \ldots, K_{n}\right)}{V\left(K_{1}, \ldots, K_{n-1}, L_{n}\right)}
$$

From Definition 1, (7), and (101), we obtain

$$
\begin{aligned}
& \frac{V_{\varphi}\left(K_{1}, \ldots, K_{n}, L_{n}\right)}{V\left(K_{1}, \ldots, K_{n-1}, L_{n}\right)}=\frac{1}{n V\left(K_{1}, \ldots, K_{n-1}, L_{n}\right)} \\
& \cdot \int_{S^{n-1}} \varphi\left(\frac{h\left(K_{n}, u\right)}{h\left(L_{n}, u\right)}\right) h\left(L_{n}, u\right) \\
& \cdot d S\left(K_{1}, \ldots, K_{n-1} ; u\right)=\frac{1}{n V\left(K_{1}, \ldots, K_{n-1}, L_{n}\right)} \\
& \quad \times \int_{S^{n-1}} \varphi\left(\frac{V\left(K_{1}, \ldots, K_{n}\right)}{V\left(K_{1}, \ldots, K_{n-1}, L_{n}\right)}\right) h\left(L_{n}, u\right) \\
& \cdot d S\left(K_{1}, \ldots, K_{n-1} ; u\right)=\varphi\left(\frac{V\left(K_{1}, \ldots, K_{n}\right)}{V\left(K_{1}, \ldots, K_{n-1}, L_{n}\right)}\right) .
\end{aligned}
$$

This implies that the equality in (97) holds. 
Theorem 17 (Orlicz-Aleksandrov-Fenchel inequality). If $K_{1}$, $\ldots, K_{n} \in \mathscr{K}_{o}^{n}, L_{n} \in \mathscr{K}_{o o}^{n}, 1 \leq r \leq n$, and $\varphi \in \Phi$, then

$$
\begin{aligned}
V_{\varphi}\left(K_{1}, \ldots, K_{n}, L_{n}\right) \\
\geq V\left(K_{1}, \ldots, K_{n-1}, L_{n}\right) \\
\quad \cdot \varphi\left(\frac{\prod_{i=1}^{r} V\left(K_{i} \ldots, K_{i}, K_{r+1}, \ldots, K_{n}\right)^{1 / r}}{V\left(K_{1}, \ldots, K_{n-1}, L_{n}\right)}\right) .
\end{aligned}
$$

Proof. This follows immediately from Lemma 16 with the Aleksandrov-Fenchel inequality.

Unfortunately, precise equality for Orlicz-AleksandrovFenchel inequality is also unknown in general, because the precise equality for the classical Aleksandrov-Fenchel inequality is unknown in general; see [38, p.408] for a full discussion.

Corollary 18. If $K_{1}, \ldots, K_{n} \in \mathscr{K}_{o}^{n}, L_{n} \in \mathscr{K}_{o o}^{n}$, and $\varphi \in \Phi$, then

$$
\begin{aligned}
V_{\varphi}( & \left.K_{1}, \ldots, K_{n}, L_{n}\right) \\
\geq & V\left(K_{1}, \ldots, K_{n-1}, L_{n}\right) \\
\quad & \varphi\left(\left(\frac{V\left(K_{1}\right) \cdots V\left(K_{n}\right)}{V\left(K_{1}, \ldots, K_{n-1}, L_{n}\right)^{n}}\right)^{1 / n}\right) .
\end{aligned}
$$

If $\varphi$ is strictly convex, equality holds if and only if $K_{1}, \ldots, K_{n}, L_{n}$ are all homothetic of each other.

Proof. This inequality (104) yields immediately from (103) with $r=n$.

Next, we discuss the equality condition of (104). When $r=n$, the Aleksandrov-Fenchel inequality becomes

$$
V\left(K_{1}, \ldots, K_{n}\right)^{n} \geq V\left(K_{1}\right) \cdots V\left(K_{n}\right) .
$$

Although, the precise equality for the Aleksandrov-Fenchel inequality are unknown in general, but it is well known that the equality in (105) holds if and only if $K_{1}, \ldots, K_{n}$ are all homothetic of each other. Hence combining the equality conditions of Lemma 16 , it follows that the equality condition of (104) holds if and only if $\varphi$ is strictly convex and $K_{1}, \ldots, K_{n}, L_{n}$ are all homothetic of each other.

Theorem 19. If $K \in \mathscr{K}_{o o}^{n}, L \in \mathscr{K}_{o}^{n}, 0 \leq i<n$, and $\varphi \in \Phi$, then

$$
W_{\varphi, i}(K, L) \geq W_{i}(K) \cdot \varphi\left(\left(\frac{W_{i}(L)}{W_{i}(K)}\right)^{1 /(n-i)}\right) .
$$

If $\varphi$ is strictly convex, equality holds if and only if $K$ and $L$ are homothetic.

Proof. This inequality (106) yields immediately from Theorem 17 with $r=n-i-1, K_{1}=\cdots=K_{n-i-1}=K, K_{n-i}=\cdots=$ $K_{n-1}=B, L_{n}=K$, and $K_{n}=L$.
Next, we discuss the equality condition of (106). When $r=n-i-1, K_{1}=\cdots=K_{n-i-1}=K, K_{n-i}=\cdots=K_{n-1}=B$, $L_{n}=K$, and $K_{n}=L$, (103) becomes

$$
\begin{aligned}
W_{\varphi, i}(K, L) \geq & W_{i}(K) \\
& \cdot \varphi\left(\frac{\left[W_{i}(K)^{n-i-1} W_{i}(L)\right]^{1 /(n-i)}}{W_{i}(K)}\right) . \\
= & W_{i}(K) \varphi\left(\left(\frac{W_{i}(L)}{W_{i}(K)}\right)^{1 /(n-i)}\right) .
\end{aligned}
$$

When $K_{1}=\cdots=K_{n-i-1}=K, K_{n-i}=\cdots=K_{n-1}=B, L_{n}=K$, and $K_{n}=L$, (97) becomes

$$
W_{\varphi, i}(K, L) \geq W_{i}(K) \cdot \varphi\left(\frac{W_{i}(K, L)}{W_{i}(K)}\right) .
$$

It is not difficult to see that we derive (107) from (109) by using the well-known inequality and with equality

$$
W_{i}(K, L)^{n-i} \geq W_{i}(K)^{n-i-1} W_{i}(L),
$$

with equality if $K$ and $L$ are all homothetic. Although, the precise equality for the Aleksandrov-Fenchel inequality are unknown in general, but combining the equality conditions of (97) and (110), it follows that the equality in (106) holds if $\varphi$ is strictly convex and if and only if $K$ and $L$ are homothetic.

Through the above discussion, it is clear that the equality in (18) (stated in the introduction) holds if and only if $K$ and $L$ are homothetic if $\varphi$ is strictly convex. Lutwak's $L_{p}$ Minkowski inequality (46) follows immediately from (106) with $\varphi(t)=t^{p}$ and $p \geq 1$.

There is a quadratic inequality including not only Minkowski first inequality (35), but also Minkowski second inequality (36). This is the celebrated quadratic AleksandrovFenchel inequality, stating that, for compact convex sets $K_{1}, \ldots, K_{n}$ in $\mathbb{R}^{n}$,

$$
\begin{aligned}
& V\left(K_{1}, K_{2}, K_{3}, \ldots, K_{n}\right)^{2} \\
& \quad \geq V\left(K_{1}, K_{1}, K_{3}, \ldots, K_{n}\right) V\left(K_{2}, K_{2}, K_{3}, \ldots, K_{n}\right) .
\end{aligned}
$$

The Aleksandrov-Fenchel inequality appears in the work of Aleksandrov [51] and Fenchel and Jessen [52] and is essentially the most powerful inequality of its type known (see [57, p.371]). Here, we give the following Orlicz quadratic Aleksandrov-Fenchel inequality.

Theorem 20 (Orlicz quadratic Aleksandrov-Fenchel inequality). If $K_{1}, \ldots, K_{n} \in \mathscr{K}_{o}^{n}, L_{n} \in \mathscr{K}_{o o}^{n}$, and $\varphi \in \Phi$, then

$$
\begin{aligned}
& V_{\varphi}\left(K_{1}, \ldots, K_{n}, L_{n}\right) \geq V\left(K_{1}, \ldots, K_{n-1}, L_{n}\right) \\
& \quad \cdot\left(\left(\frac{V\left(K_{1}, K_{1}, K_{3}, \ldots, K_{n}\right) V\left(K_{2}, K_{2}, K_{3}, \ldots, K_{n}\right)}{V\left(K_{1}, \ldots, K_{n-1}, L_{n}\right)^{2}}\right)^{1 / 2}\right) .
\end{aligned}
$$

Proof. This follows immediately from (103). 
Obviously, when $\varphi(t)=t^{p}$ and $p=1$, (112) becomes (111). On the other hand, putting $L_{n}=K_{n}$ and combining $\phi$ which is increasing function in (112), (112) becomes also (111).

Theorem 21 (Orlicz isoperimetric inequality). If $K \in \mathscr{K}_{o}^{n}$, $\varphi \in \Phi$, and $0 \leq i<n$, then

$$
\begin{gathered}
\frac{V_{\varphi}(K[n-i-1], B[i+1], K)}{W_{i}(K)} \\
\geq \varphi\left(\left(\frac{\kappa_{n}}{W_{i}(K)}\right)^{1 /(n-i)}\right) .
\end{gathered}
$$

If $\varphi$ is strictly convex, it equality holds if and only if $K$ is a ball.

Proof. This follows immediately from (103) with $r=n-i-1$, $K_{1}=\cdots=K_{n-i-1}=K, K_{n-i}=\cdots=K_{n-1}=B, L_{n}=K$, and $K_{n}=B$.

When $\varphi(t)=t^{p}, p=1$, and $i=0$, Orlicz isoperimetric inequality (113) becomes the classical isoperimetric inequality. If $K$ is convex body, then (see, e.g., [50, p.382])

$$
\left(\frac{S(K)}{\omega_{n}}\right)^{n} \geq\left(\frac{V(K)}{\kappa_{n}}\right)^{n-1}
$$

with equality if and only if $K$ is ball.

The well-known Urysohn's inequality states that if $K$ is convex body, then

$$
\left(\frac{w(K)}{2}\right)^{n} \geq \frac{V(K)}{\kappa_{n}}
$$

with equality if and only if $K$ is ball (see [50, p.382]). Here, $w(K)$ is the mean width of convex body $K$, denoted by

$$
w(K):=\frac{2}{\kappa_{n}} W_{n-1}(K)=\frac{2}{\kappa_{n}} V(K, B, \ldots, B) .
$$

In the following, we establish an Orlicz Urysohn's inequality.

Theorem 22 (Orlicz Urysohn's inequality). If $K \in \mathscr{K}_{0}^{n}, \varphi \in$ $\Phi$, and $0 \leq i<n$, then

$$
\frac{V_{\varphi}(B[n-1], K, B)}{\kappa_{n}} \geq \varphi\left(\left(\frac{W_{i}(K)}{\kappa_{n}}\right)^{1 /(n-i)}\right) .
$$

If $\varphi$ is strictly convex, equality holds if and only if $K$ is a ball.

Proof. This follows immediately from (103) with $r=n-i-1$, $K_{1}=\cdots=K_{n-1}=B, L_{n}=B$, and $K_{n}=K$.

When $\varphi(t)=t^{p}$ and $p \geq 1$, the Orlicz Urysohn's inequality becomes the following $L_{p}$-Urysohn's inequality. If $K \in \mathscr{K}_{o}^{n}$ and $p \geq 1$ and $0 \leq i<n$, then

$$
\left(\frac{W_{p, i}(B, K)}{\kappa_{n}}\right)^{(n-i) / p} \geq \frac{W_{i}(K)}{\kappa_{n}} .
$$

If $\varphi$ is strictly convex, equality holds if and only if $K$ is a ball. Putting $p=1$ and $i=0$ in (118), (118) becomes the Urysohn's inequality (115).

The following uniqueness is a direct consequence of the Orlicz-Aleksandrov-Fenchel inequality for the Orlicz multiple mixed volumes.

Theorem 23. If $K_{1}, \ldots, K_{n}, L_{n} \in \mathscr{M} \subset \mathscr{K}_{o}^{n}$, and $\varphi \in \Phi$ are strictly convex and if either

$$
\begin{array}{r}
V_{\varphi}\left(K_{1}, \ldots, K_{n-1}, L_{n}, Q\right)=V_{\varphi}\left(K_{1}, \ldots, K_{n-1}, K_{n}, Q\right), \\
\text { for all } Q \in \mathscr{M},
\end{array}
$$

or

$$
\begin{aligned}
& \frac{V_{\varphi}\left(K_{1}, \ldots, K_{n-1}, Q, L_{n}\right)}{V\left(K_{1}, \ldots, K_{n-1}, L_{n}\right)} \\
& \quad=\frac{V_{\varphi}\left(K_{1}, \ldots, K_{n-1}, Q, K_{n}\right)}{V\left(K_{1}, \ldots, K_{n}\right)}, \quad \text { for all } Q \in \mathscr{M},
\end{aligned}
$$

then $K_{n}=L_{n}$.

Proof. Suppose (119) hold. Taking $L_{n}$ for $Q$, then from Definition 1 and Lemma 16, we obtain

$$
\begin{gathered}
V\left(K_{1}, \ldots, K_{n-1}, L_{n}\right)=V_{\varphi}\left(K_{1}, \ldots, K_{n}, L_{n}\right) \\
\geq V\left(K_{1}, \ldots, K_{n-1}, L_{n}\right) \\
\cdot \varphi\left(\left(\frac{V\left(K_{1}, \ldots, K_{n}\right)}{V\left(K_{1}, \ldots, K_{n-1}, L_{n}\right)}\right)\right)
\end{gathered}
$$

with equality if and only if $K_{n}$ and $L_{n}$ are homothetic. Hence

$$
1 \geq \varphi\left(\frac{V\left(K_{1}, \ldots, K_{n}\right)}{V\left(K_{1}, \ldots, K_{n-1}, L_{n}\right)}\right),
$$

with equality if and only if $K_{n}$ and $L_{n}$ are homothetic. Since $\varphi$ is increasing function on $(0, \infty)$, this follows that

$$
V\left(K_{1}, \ldots, K_{n}\right) \leq V\left(K_{1}, \ldots, K_{n-1}, L_{n}\right),
$$

with equality if and only if $K_{n}$ and $L_{n}$ are homothetic. On the other hand, if taking $K_{n}$ for $Q$, we similarly get $V\left(K_{1}, \ldots, K_{n}\right) \geq V\left(K_{1}, \ldots, K_{n-1}, L_{n}\right)$, with equality if and only if $K_{n}$ and $L_{n}$ are homothetic. Hence $V\left(K_{1}, \ldots, K_{n}\right)=$ $V\left(K_{1}, \ldots, K_{n-1}, L_{n}\right)$, and $K_{n}$ and $L_{n}$ are homothetic; it follows that $K_{n}$ and $L_{n}$ must be equal.

Suppose (120) hold. Taking $K_{n}$ for $Q$, then from Definition 1 and Lemma 16, we obtain

$$
\begin{aligned}
1 & =\frac{V_{\varphi}\left(K_{1}, \ldots, K_{n}, L_{n}\right)}{V\left(K_{1}, \ldots, K_{n-1}, L_{n}\right)} \\
& \geq \varphi\left(\frac{V\left(K_{1}, \ldots, K_{n}\right)}{V\left(K_{1}, \ldots, K_{n-1}, L_{n}\right)}\right),
\end{aligned}
$$

with equality if and only if $K_{n}$ and $L_{n}$ are homothetic. Since $\varphi$ is increasing function on $(0, \infty)$, this follows that

$$
V\left(K_{1}, \ldots, K_{n-1}, L_{n}\right) \geq V\left(K_{1}, \ldots, K_{n}\right),
$$


with equality if and only if $K_{n}$ and $L_{n}$ are homothetic. On the other hand, if taking $L_{n}$ for $Q$, we similarly get $V\left(K_{1}, \ldots, K_{n-1}, L_{n}\right) \leq V\left(K_{1}, \ldots, K_{n}\right)$, with equality if and only if $K_{n}$ and $L_{n}$ are homothetic. Hence $V\left(K_{1}, \ldots, K_{n-1}, L_{n}\right)=V\left(K_{1}, \ldots, K_{n}\right)$, and $K_{n}$ and $L_{n}$ are homothetic; it follows that $K_{n}$ and $L_{n}$ must be equal.

Corollary 24. Let $K, L \in \mathscr{M} \subset \mathscr{K}_{o}^{n}, 0 \leq i<n$, and $\varphi \in \Phi$ be strictly convex, and if either

$$
W_{\varphi, i}(Q, K)=W_{\varphi, i}(Q, L), \quad \text { for all } Q \in \mathscr{M}
$$

or

$$
\frac{W_{\varphi, i}(K, Q)}{W_{i}(K)}=\frac{W_{\varphi, i}(L, Q)}{W_{i}(L)}, \quad \text { for all } Q \in \mathscr{M}
$$

then $K=L$.

Proof. This yields immediately from Theorem 23 and Lemma 12.

\section{Orlicz-Brunn-Minkowski Inequality for Mixed Volumes}

Theorem 25 (Orlicz-Brunn-Minkowski inequality). If $K_{1}$, $\ldots, K_{n}, L_{n} \in \mathscr{K}_{o}^{n}$, and $\varphi \in \Phi$, then for $\varepsilon>0$

$$
\begin{gathered}
1 \geq \varphi\left(\frac{V\left(K_{1}, \ldots, K_{n}\right)}{V\left(K_{1}, \ldots, K_{n-1}, K_{n}+_{\varphi, \varepsilon} L_{n}\right)}\right)+\varepsilon \\
\cdot \varphi\left(\frac{V\left(K_{1}, \ldots, K_{n-1}, L_{n}\right)}{V\left(K_{1}, \ldots, K_{n-1}, K_{n}+{ }_{\varphi, \varepsilon} L_{n}\right)}\right),
\end{gathered}
$$

If $\varphi$ is strictly convex, equality holds if and only if $K_{n}$ and $L_{n}$ are homothetic.

Proof. Suppose $K_{n}, L_{n} \in \mathscr{K}_{o}^{n}$, and let

$$
Q=K_{n}+{ }_{\varphi, \varepsilon} L_{n}
$$

From Definition 1, (7), (48), and (51), and Lemma 16, we obtain

$$
\begin{aligned}
& V\left(K_{1}, \ldots, K_{n-1}, Q\right)=\frac{1}{n} \\
& \cdot \int_{S^{n-1}} \varphi\left(\frac{h\left(K_{n}, u\right)}{h(Q, u)}, \frac{h\left(L_{n}, u\right)}{h(Q, u)}\right) h(Q, u) \\
& \cdot d S\left(K_{1}, \ldots, K_{n-1} ; u\right)=\frac{1}{n} \\
& \cdot \int_{S^{n-1}}\left(\varphi\left(\frac{h\left(K_{n}, u\right)}{h(Q, u)}\right)+\varepsilon \cdot \varphi\left(\frac{h\left(L_{n}, u\right)}{h(Q, u)}\right)\right) \\
& \cdot h(Q, u) d S\left(K_{1}, \ldots, K_{n-1} ; u\right)
\end{aligned}
$$

$$
\begin{aligned}
& =V_{\varphi}\left(K_{1}, \ldots, K_{n}, Q\right)+\varepsilon \cdot V_{\varphi}\left(K_{1}, \ldots, K_{n-1}, L_{n}, Q\right) \\
& \geq V\left(K_{1}, \ldots, K_{n-1}, Q\right)\left\{\varphi\left(\frac{V\left(K_{1}, \ldots, K_{n}\right)}{V\left(K_{1}, \ldots, K_{n-1}, Q\right)}\right)\right. \\
& \left.+\varepsilon \cdot \varphi\left(\frac{V\left(K_{1}, \ldots, K_{n-1}, L_{n}\right)}{V\left(K_{1}, \ldots, K_{n-1}, Q\right)}\right)\right\} .
\end{aligned}
$$

Taking Q for $K_{n}+{ }_{\varphi} L_{n}$ in (130), (128) follows.

From the equality condition of Lemma 16, the equality in (130) holds if and only if $K_{n}$ and $Q$ are homothetic, and $L_{n}$ and $Q$ are homothetic. This yields that if $\varphi$ is strictly convex, the equality in (130) holds if and only if $K_{n}$ and $L_{n}$ are homothetic.

Corollary 26. If $K_{1}, \ldots, K_{n}, L_{n} \in \mathscr{K}_{o}^{n}, 0 \leq i \leq n, 1 \leq r \leq n$, and $\varphi \in \Phi$, then for $\varepsilon>0$

$$
\begin{aligned}
1 \geq & \varphi\left(\frac{\prod_{i=1}^{r} V\left(K_{i}, \ldots, K_{i}, K_{r+1}, \ldots, K_{n}\right)^{1 / r}}{V\left(K_{1}, \ldots, K_{n-1}, K_{n}{ }_{\varphi, \varepsilon} L_{n}\right)}\right)+\varepsilon \\
& \cdot \varphi\left(\frac{\prod_{i=1, i \neq n}^{r} V\left(K_{i}, \ldots, K_{i}, K_{r+1}, \ldots, K_{n-1}, L_{n}\right)^{1 / r}}{V\left(K_{1}, \ldots, K_{n-1}, K_{n}+_{\varphi, \varepsilon} L_{n}\right)}\right) .
\end{aligned}
$$

Proof. This follows immediately from Theorem 25 combining the Aleksandrov-Fenchel inequality.

Corollary 27. If $K_{1}, \ldots, K_{n}, L_{n} \in \mathscr{K}_{o}^{n}$, and $\varphi \in \Phi$, then for $\varepsilon>0$

$$
\begin{gathered}
1 \geq \varphi\left(\left(\frac{V\left(K_{1}\right) \cdots V\left(K_{n}\right)}{V\left(K_{1}, \ldots, K_{n-1}, K_{n}{ }_{\varphi, \varepsilon} L_{n}\right)^{n}}\right)^{1 / n}\right)+\varepsilon \\
\cdot\left(\left(\frac{V\left(K_{1}\right) \cdots V\left(K_{n-1}\right) V\left(L_{n}\right)}{V\left(K_{1}, \ldots, K_{n-1}, K_{n}{ }{ }_{\varphi, \varepsilon} L_{n}\right)^{n}}\right)^{1 / n}\right) .
\end{gathered}
$$

If $\varphi$ is strictly convex, equality holds if and only if $K_{1}, \ldots, K_{n}, L_{n}$ are all homothetic of each other.

Proof. This inequality (132) follows immediately from (131) with $r=n$.

Next, we discuss the equality condition of (132). The precise equality for the Aleksandrov-Fenchel inequality is unknown in general, but the following inequality with equality is clear:

$$
V\left(K_{1}, \ldots, K_{n}\right)^{n} \geq V\left(K_{1}\right) \cdots V\left(K_{n}\right),
$$

with equality if and only if $K_{1}, \ldots, K_{n}$ are all homothetic of each other. Hence combining the equality conditions of (128) and (133), it follows that the equality in (132) holds if $\varphi$ is strictly convex and if and only if $K_{1}, \ldots, K_{n}, L_{n}$ are all homothetic of each other. 
Corollary 28. If $K, L \in \mathscr{K}_{o}^{n}, \varphi \in \Phi$, and $0 \leq i<n$, then for $\varepsilon>0$

$$
\begin{gathered}
1 \geq \varphi\left(\left(\frac{W_{i}(K)}{W_{i}\left(K+_{\varphi, \varepsilon} L\right)}\right)^{1 /(n-i)}\right)+\varepsilon \\
\cdot \varphi\left(\left(\frac{W_{i}(L)}{W_{i}\left(K+_{\varphi, \varepsilon} L\right)}\right)^{1 /(n-i)}\right) .
\end{gathered}
$$

If $\varphi$ is strictly convex, equality holds if and only if $K$ and $L$ are homothetic.

Proof. This inequality (134) follows immediately from Corollary 26 with $r=n-i-1, K_{1}=\cdots=K_{n-i-1}=K+_{\varphi, \varepsilon} L K_{n-i}=$ $\cdots=K_{n-1}=B, K_{n}=K$, and $L_{n}=L$.

Next, we discuss the equality condition of (134). On the one hand, putting $r=n-i-1, K_{1}=\cdots=K_{n-i-1}=$ $K+_{\varphi, \varepsilon} L K_{n-i}=\cdots=K_{n-1}=B, K_{n}=K$, and $L_{n}=L$ in Theorem 25, we have

$$
\begin{array}{r}
1 \geq \varphi\left(\left(\frac{W_{i}(K)}{W_{i}\left(K+_{\varphi, \varepsilon} L\right)}\right)^{1 /(n-i)}\right)+\varepsilon \\
\cdot \varphi\left(\left(\frac{W_{i}(L)}{W_{i}\left(K+_{\varphi, \varepsilon} L\right)}\right)^{1 /(n-i)}\right) .
\end{array}
$$

On the other hand, putting $K_{1}=\cdots=K_{n-i-1}=$ $K+_{\varphi, \varepsilon} L K_{n-i}=\cdots=K_{n-1}=B, K_{n}=K$, and $L_{n}=L$ in Theorem 25, we have

$$
\begin{gathered}
1 \geq \varphi\left(\frac{W_{i}\left(K+_{\varphi, \varepsilon} L, K\right)}{W_{i}\left(K+_{\varphi, \varepsilon} L\right)}\right)+\varepsilon \\
\cdot \varphi\left(\frac{W_{i}\left(K+_{\varphi, \varepsilon} L, L\right)}{W_{i}\left(K+_{\varphi, \varepsilon} L\right)}\right),
\end{gathered}
$$

It is not difficult to see that we derive (135) from (136) by using the following inequalities and with equalities:

$$
W_{i}\left(K+_{\varphi, \varepsilon} L, K\right)^{n-i} \geq W_{i}\left(K+_{\varphi, \varepsilon} L\right)^{n-i-1} W_{i}(K),
$$

with equality if $K$ and $K{ }_{\varphi, \varepsilon} L$ are all homothetic, and

$$
W_{i}\left(K+_{\varphi, \varepsilon} L, L\right)^{n-i} \geq W_{i}\left(K+_{\varphi, \varepsilon} L\right)^{n-i-1} W_{i}(L),
$$

with equality if $K+_{\varphi, \varepsilon} L$ and $L$ are all homothetic. Although, the precise equality for the Aleksandrov-Fenchel inequality are unknown in general, but combining the equality conditions of (128), (137), and (138), we obtain that the equality in (134) holds if $\varphi$ is strictly convex, if and only if $K$ and $L$ are homothetic, $K$ and $K+_{\varphi, \varepsilon} L$ are homothetic, and $K+_{\varphi, \varepsilon} L$ and $L$ are homothetic, this shows that the equality in (134) holds if and only if $K$ and $L$ are homothetic, if $\varphi$ is strictly convex.
Theorem $29\left(L_{p}\right.$-Brunn-Minkowski inequality for the mixed volumes). If $K_{1}, \ldots, K_{n}, L_{n} \in \mathscr{K}_{0}^{n}, 0 \leq i<n, 1 \leq r \leq n$, and $p \geq 1$, then

$$
\begin{aligned}
V( & \left.K_{1}, \ldots, K_{n-1}, K_{n}+{ }_{p} L_{n}\right)^{p} \\
\geq & \prod_{i=1}^{r} V\left(K_{i}, \ldots, K_{i}, K_{r+1}, \ldots, K_{n}\right)^{p / r} \\
& \quad+\prod_{i=1, i \neq n}^{r} V\left(K_{i}, \ldots, K_{i}, K_{r+1}, \ldots, K_{n-1}, L_{n}\right)^{p / r},
\end{aligned}
$$

Proof. This follows immediately from Corollary 26 with $\varphi(t)=t^{p}$ and $p \geq 1$.

Putting $r=n-i-1, K_{1}=\cdots=K_{n-i-1}=K{ }_{p} L, K_{n-i}=$ $\cdots=K_{n-1}=B, K_{n}=K$, and $L_{n}=L$ in (139), (139) becomes also Lutwak's $L_{p}$-Brunn-Minkowski inequality (47). Putting $r=n$ in (139), we get the following interesting result:

$$
\begin{aligned}
V\left(K_{1} \cdots K_{n-1}, K_{n}+{ }_{p} L_{n}\right)^{p} \\
\geq \\
\quad\left(V\left(K_{1}\right) \cdots V\left(K_{n}\right)\right)^{p / n} \\
\quad+\left(V\left(K_{1}\right) \cdots V\left(K_{n-1}\right) V\left(L_{n}\right)\right)^{p / n} .
\end{aligned}
$$

with equality if and only if $K_{1}, \ldots, K_{n}, L_{n}$ are all homothetic of each other.

Corollary 30. If $K_{1}, \ldots, K_{n} \in \mathscr{K}_{o}^{n}, L_{n} \in \mathscr{K}_{o o}^{\mathrm{n}}, 1 \leq r \leq n$, and $\varphi \in \Phi$, then

$$
\begin{aligned}
V_{\varphi}( & \left.K_{1}, \ldots, K_{n}, L_{n}\right) \\
\geq & V\left(K_{1}, \ldots, K_{n-1}, L_{n}\right) \\
& \cdot \varphi\left(\frac{\prod_{i=1}^{r} V\left(K_{i} \ldots, K_{i}, K_{r+1}, \ldots, K_{n}\right)^{1 / r}}{V\left(K_{1}, \ldots, K_{n-1}, L_{n}\right)}\right) .
\end{aligned}
$$

Proof. Let

$$
K_{\varepsilon}=L_{n}+{ }_{\varphi, \varepsilon} K_{n} .
$$

From (8), (69), and (131), we obtain

$$
\begin{aligned}
\frac{1}{\varphi_{l}^{\prime}(1)} \cdot V_{\varphi}\left(K_{1}, \ldots, K_{n}, L_{n}\right)=\left.\frac{d}{d \varepsilon}\right|_{\varepsilon=0^{+}} V\left(K_{1}, \ldots, K_{n-1}, K_{\varepsilon}\right) \\
=\lim _{\varepsilon \rightarrow 0^{+}} \frac{V\left(K_{1}, \ldots, K_{n-1}, K_{\varepsilon}\right)-V\left(K_{1}, \ldots, K_{n-1}, L_{n}\right)}{\varepsilon} \\
=\lim _{\varepsilon \rightarrow 0^{+}} \frac{1-V\left(K_{1}, \ldots, K_{n-1}, L_{n}\right) / V\left(K_{1}, \ldots, K_{n-1}, K_{\varepsilon}\right)}{\left.\left.1-K_{1}, \ldots, K_{n-1}, L_{n}\right) / V\left(K_{1}, \ldots, K_{n-1}, K_{\varepsilon}\right)\right)} \\
\quad \cdot \frac{1-\varphi\left(V\left(K_{1}, \ldots, K_{n-1}, L_{n}\right) / V\left(K_{1}, \ldots, K_{n-1}, K_{\varepsilon}\right)\right)}{\varepsilon} \\
\cdot V\left(K_{1}, \ldots, K_{n-1}, K_{\varepsilon}\right)
\end{aligned}
$$




$$
\begin{aligned}
= & \lim _{t \rightarrow 0^{+}} \frac{1-t}{\varphi(1)-\varphi_{1}(t)} \\
& \cdot \lim _{\varepsilon \rightarrow 0^{+}} \frac{1-\varphi\left(V\left(K_{1}, \ldots, K_{n-1}, L_{n}\right) / V\left(K_{1}, \ldots, K_{n-1}, K_{\varepsilon}\right)\right)}{\varepsilon} \\
& \cdot \lim _{\varepsilon \rightarrow 0^{+}} V\left(K_{1}, \ldots, K_{n-1}, K_{\varepsilon}\right) \\
\geq & \frac{1}{\varphi_{l}^{\prime}(1)} \cdot \varphi\left(\frac{V\left(K_{1}, \ldots, K_{n-1}, K_{n}\right)}{V\left(K_{1}, \ldots, K_{n-1}, L_{n}\right)}\right) \cdot V\left(K_{1}, \ldots, K_{n-1}, L_{n}\right) \\
\geq & \frac{1}{\varphi_{l}^{\prime}(1)} \cdot \varphi\left(\frac{\prod_{i=1}^{r}\left(K_{i}, \ldots, K_{i}, K_{r+1}, \ldots, K_{n}\right)^{1 / r}}{V\left(K_{1}, \ldots, K_{n-1}, L_{n}\right)}\right) \\
& \cdot V\left(K_{1}, \ldots, K_{n-1}, L_{n}\right) .
\end{aligned}
$$

From (143), (141) easy follows. This proof is complete.

Finally, it is worth mentioning that we may see that the Orlicz-Aleksandrov-Fenchel inequality for the Orlicz multiple mixed volumes implies Orlicz-Brunn-Minkowski inequality for the mixed volumes, from the proof of Theorem 25. This combines Corollary 30, it yields that the OrliczAleksandrov-Fenchel inequality is equivalent to the OrliczBrunn-Minkowski inequality.

\section{Data Availability}

The data used to support the findings of this study are included within the article.

\section{Conflicts of Interest}

The author declares that he has no conflicts of interest.

\section{Authors' Contributions}

Chang-Jian Zhao provided the questions and gave the proof for the all results.

\section{Acknowledgments}

The author expresses his thanks to Professors G. Leng and W. $\mathrm{Li}$ for their valuable help. The author's research is supported by National Natural Science Foundation of China (11371334 and 10971205).

\section{References}

[1] W. J. Firey, "Polar means of convex bodies and a dual to the Brunn-Minkowski theorem," Canadian Journal of Mathematics. Journal Canadien de Mathematiques, vol. 13, pp. 444-453, 1961.

[2] W. J. Firey, "p-Means of convex bodies," Mathematica Scandinavica, vol. 10, pp. 17-24, 1962.

[3] S. Campi and P. Gronchi, "The Lp-Busemann-Petty centroid inequality," Advances in Mathematics, vol. 167, no. 1, pp. 128-141, 2002.

[4] S. Campi and P. Gronchi, "On the reverse Lp-Busemann-Petty centroid inequality," Mathematika, vol. 49, no. 1-2, pp. 1-11, 2002.
[5] S. Campi and P. Gronchi, "Volume inequalities for Lpzonotopes," Mathematika, vol. 53, no. 1, pp. 71-80, 2006.

[6] K.-S. Chou and X.-J. Wang, "The Lp-Minkowski problem and the Minkowski problem in centroaffine geometry," Advances in Mathematics, vol. 205, no. 1, pp. 33-83, 2006.

[7] C. Haberl and L. Parapatits, "The centro-affine Hadwiger theorem," Journal of American Mathematical Society, vol. 27, no. 3, pp. 685-705, 2014.

[8] C. Haberl and F. E. Schuster, "Asymmetric affine Lp Sobolev inequalities," Journal of Functional Analysis, vol. 257, no. 3, pp. 641-658, 2009.

[9] C. Haberl and F. E. Schuster, "General $\mathrm{L}_{p}$ affine isoperimetric inequalities," Journal of Differential Geometry, vol. 83, no. 1, pp. 1-26, 2009.

[10] C. Haberl, F. Schuster, and J. Xiao, "An asymmetric affine PólyaSzegö principle," Mathematische Annalen, vol. 352, no. 3, pp. 517-542, 2012

[11] M. Ludwig and M. Reitzner, "A classification of SL(n) invariant valuations," Annals of Mathematics, vol. 172, no. 2, pp. 1219-1267, 2010.

[12] E. Lutwak, "The Brunn-Minkowski-Firey theory II. Affine and geominimal surface areas," Advances in Mathematics, vol. 118, no. 2, pp. 244-294, 1996.

[13] E. Lutwak, D. Yang, and G. Zhang, "On the $\mathrm{L}_{p}$-Minkowski problem," Transactions of American Mathematical Society, vol. 356, no. 11, pp. 4359-4370, 2004.

[14] E. Lutwak, D. Yang, and G. Zhang, "Lp John ellipsoids," Proceedings of London Mathematical Society, vol. 90, no. 2, pp. 497-520, 2005.

[15] E. Lutwak, D. Yang, and G. Zhang, "Lp affine isoperimetric inequalities," Journal of Differential Geometry, vol. 56, no. 1, pp. 111-132, 2000.

[16] E. Lutwak, D. Yang, and G. Zhang, "Sharp affine Lp Sobolev inequalities," Journal of Differential Geometry, vol. 62, no. 1, pp. $17-38,2002$.

[17] E. Lutwak, D. Yang, and G. Zhang, "The Brunn-MinkowskiFirey inequality for nonconvex sets," Advances in Applied Mathematics, vol. 48, no. 2, pp. 407-413, 2012.

[18] L. Parapatits, "SL(n)-Covariant Lp-Minkowski Valuations," Journal Of London Mathematical Society, vol. 89, no. 2, pp. 397414, 2014.

[19] L. Parapatits, "SL(n)-Contravariant Lp-Minkowski Valuations," Transactions of American Mathematical Society, vol. 366, no. 3, pp. 1195-1211, 2014.

[20] C. Schutt and E. Werner, "Surface bodies and p-affine surface area," Advances in Mathematics, vol. 187, pp. 98-145, 2004.

[21] E. Werner, "Rényi divergence and Lp-affine surface area for convex bodies," Advances in Mathematics, vol. 230, no. 3, pp. 1040-1059, 2012.

[22] E. Werner and D. Ye, "New Lp affine isoperimetric inequalities," Advances in Mathematics, vol. 218, no. 3, pp. 762-780, 2008.

[23] E. Lutwak, D. Yang, and G. Zhang, "Orlicz projection bodies," Advances in Mathematics, vol. 223, no. 1, pp. 220-242, 2010.

[24] E. Lutwak, D. Yang, and G. Zhang, "Orlicz centroid bodies," Journal of Differential Geometry, vol. 84, no. 2, pp. 365-387, 2010.

[25] R. J. Gardner, D. Hug, and W. Weil, "The Orlicz-BrunnMinkowski theory: a general framework, additions, and inequalities," Journal of Differential Geometry, vol. 97, no. 3, pp. 427-476, 2014. 
[26] D. Xi, H. Jin, and G. Leng, "The Orlicz Brunn-Minkowski inequality," Advances in Mathematics, vol. 260, pp. 350-374, 2014.

[27] G. Zhu, "The Orlicz centroid inequality for star bodies," Advances in Applied Mathematics, vol. 48, no. 2, pp. 432-445, 2012.

[28] C. Haberl, E. Lutwak, D. Yang, and G. Zhang, "The even Orlicz Minkowski problem," Advances in Mathematics, vol. 224, no. 6, pp. 2485-2510, 2010.

[29] M. Ludwig, "General affine surface areas," Advances in Mathematics, vol. 224, no. 6, pp. 2346-2360, 2010.

[30] D. Ye, "Inequalities for general mixed affine surface areas," Journal Of London Mathematical Society-Second Series, vol. 85, no. 1, pp. 101-120, 2012.

[31] U. Caglar and D. Ye, "Affine isoperimetric inequalities in the functional Orlicz-Brunn-Minkowski theory," Advances in Applied Mathematics, vol. 81, pp. 78-114, 2016.

[32] F. Chen and G. Leng, "Orlicz-Brunn-Minkowski inequalities for Blaschke-Minkowski homomorphisms," Geometriae Dedicata, vol. 187, pp. 137-149, 2017.

[33] Q. Huang and B. He, "On the Orlicz Minkowski Problem for Polytopes," Discrete \& Computational Geometry, vol. 48, no. 2, pp. 281-297, 2012.

[34] H. Jin, S. Yuan, and G. Leng, "On the dual Orlicz mixed volumes," Chinese Annals of Mathematics, Series B, vol. 36, no. 6, pp. 1019-1026, 2015.

[35] M. A. Krasnosel'skil and J. B. Rutickil, Convex functions and Orlicz spaces, P. Noordhoff Ltd., Groningen, Netherlands, 1961.

[36] A.-J. Li and G. Leng, "A new proof of the Orlicz Busemann-Petty centroid inequality," Proceedings of American Mathematical Society, vol. 139, no. 4, pp. 1473-1481, 2011.

[37] Y. Lin, "Affine Orlicz Pólya-Szegö principle for log-concave functions," Journal of Functional Analysis, vol. 273, no. 10, pp. 3295-3326, 2017.

[38] M. M. Rao and Z. D. Ren, Theory of Orlicz Spaces, Marcel Dekker, New York, NY, USA, 1991.

[39] Y. Sun and Y. Long, "The planar Orlicz Minkowski problem in the L1-sense," Advances in Mathematics, vol. 281, pp. 1364-1383, 2015.

[40] W. Wang, W. Shi, and S. Ye, "Dual mixed Orlicz-BrunnMinkowski inequality and dual Orlicz mixed quermassintegrals," Indagationes Mathematicae. New Series, vol. 28, no. 4, pp. 721-735, 2017.

[41] D. Xi and G. Leng, "Dar's conjecture and the log-BrunnMinkowski inequality," Journal of Differential Geometry, vol. 103, no. 1, pp. 145-189, 2016.

[42] C.-J. Zhao, "Orlicz dual mixed volumes," Results in Mathematics, vol. 68 , no. 1-2, pp. 93-104, 2015.

[43] C. J. Zhao, "On the Orlicz-Brunn-Minkowski theory," Balkan Journal of Geometry and Its Applications, vol. 22, no. 1, pp. 98121, 2017.

[44] C.-J. Zhao, "Orlicz-Brunn-Minkowski inequality for radial Blaschke-Minkowski homomorphisms," Quaestiones Mathematicae, 2018.

[45] C.-J. Zhao, "Orlicz dual affine quermassintegrals," Forum Mathematicum, vol. 30, no. 4, pp. 929-945, 2018.

[46] C.-J. Zhao and W. S. Cheung, "Orlicz mixed affine quermassintegrals," Balkan Journal of Geometry and Its Applications, vol. 23, pp. 76-96, 2018.
[47] C.-J. Zhao and W. S. Cheung, "Orlicz Mean Dual Affine Quermassintegrals," Journal of Function Spaces, vol. 2018, Article ID 8123924, 13 pages, 2018.

[48] B. Zhu, J. Zhou, and W. Xu, "Dual Orlicz-Brunn-Minkowski theory," Advances in Mathematics, vol. 264, pp. 700-725, 2014.

[49] E. Lutwak, "The Brunn-Minkowski-Firey theory. I. Mixed volumes and the Minkowski problem," Journal of Differential Geometry, vol. 38, no. 1, pp. 131-150, 1993.

[50] R. Schneider, Convex Bodies: the Brunn-Minkowski theory, Cambridge University Press, Cambridge, UK, 2014.

[51] A. D. Aleksandrov, "On the theory of mixed volumes. I. Extension of certain concepts in the theory of convex bodies," Matematicheskii Sbornik, vol. 2, pp. 947-972, 1937.

[52] W. Fenchel and B. Jessen, Theorie der Konvexen Körper, Springer Berlin Heidelberg, Berlin, Heidelberg, 1934.

[53] Y. D. Burago and V. A. Zalgaller, Geometric Inequalities, vol. 285, Springer-Verlag, Berlin, Germany, 1988.

[54] H. Busemann, Convex Surfaces, Interscience, New York, USA, 1958.

[55] R. Schneider, "Boundary structure and curvature of convex bodies," in Contributions to Geometry, pp. 13-59, Birkhäuser, Basel, Switzerland, 1979.

[56] K. Leichtweiss, Konvexe Mengen, Springer-Verlag, Berlin-New York, 1980.

[57] R. J. Gardner, Geometric Tomography, Cambridge University Press, New York, USA, 2nd edition, 2006.

[58] E. Lutwak, "Mixed projection inequalities," Transactions of American Mathematical Society, vol. 287, no. 1, pp. 91-105, 1985.

[59] E. Lutwak, "Volume of mixed bodies," Transactions of American Mathematical Society, vol. 294, no. 2, pp. 487-500, 1986.

[60] J. Hoffmann-Jorgensen, Probability With a View Toward Statistics, vol. I, Chapman and Hall, New York, USA, 1994. 


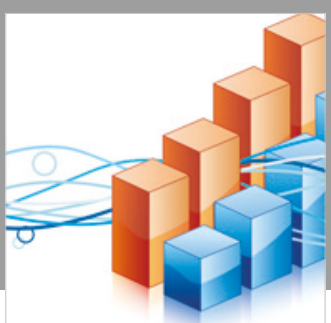

Advances in

Operations Research

\section{-n-m}
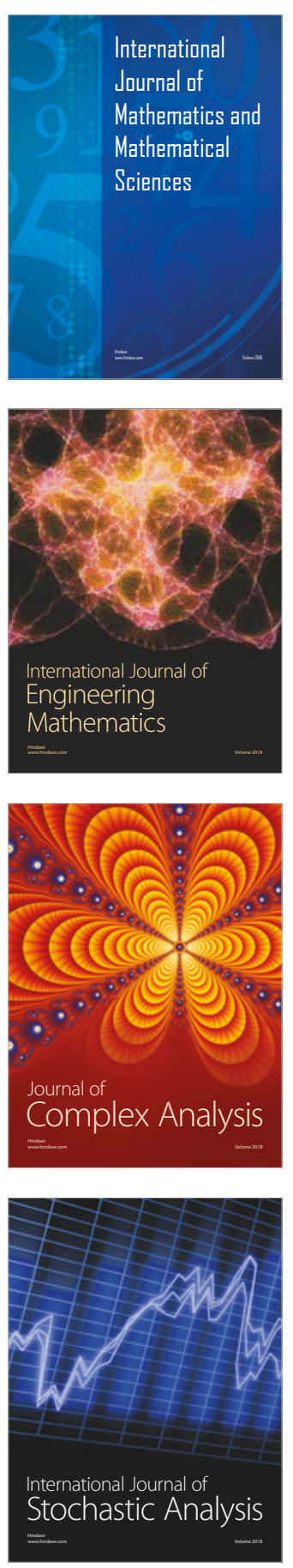
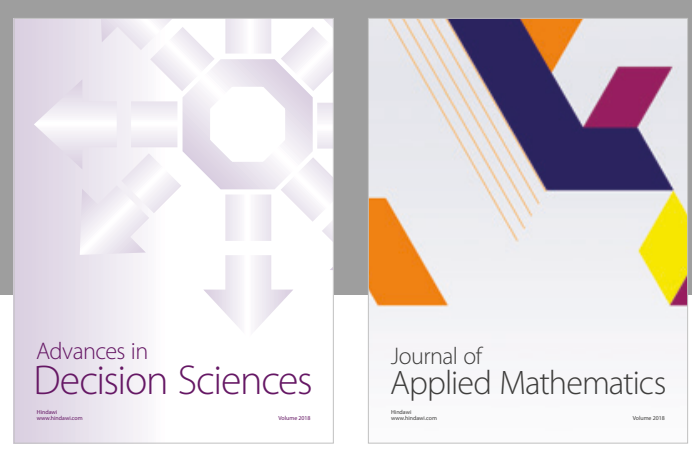

Journal of

Applied Mathematics
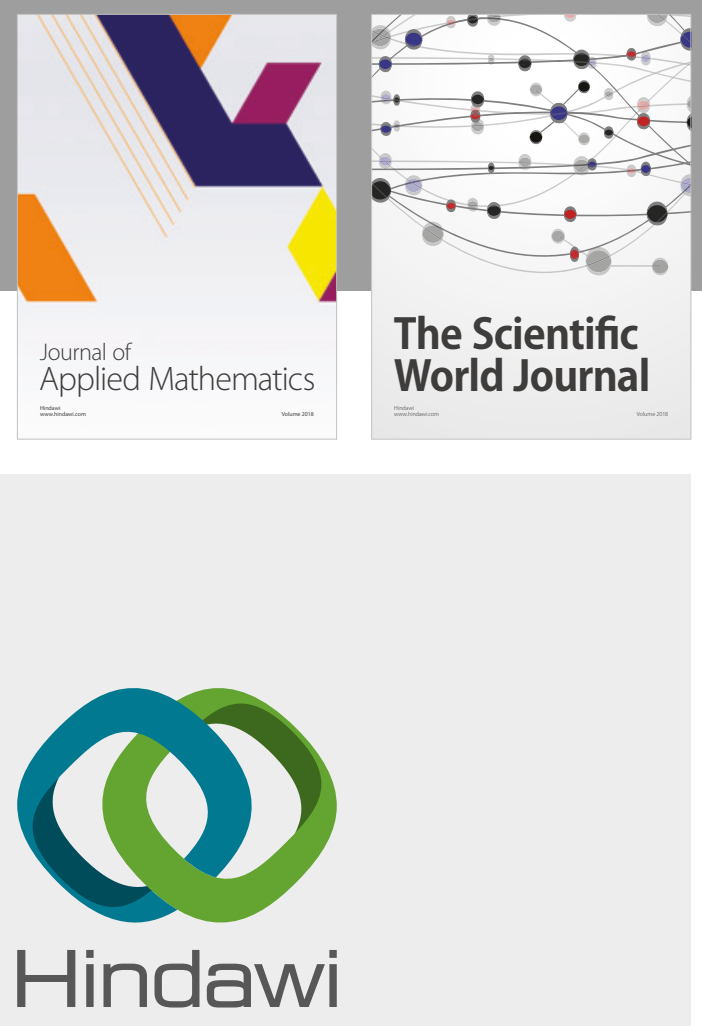

Submit your manuscripts at

www.hindawi.com

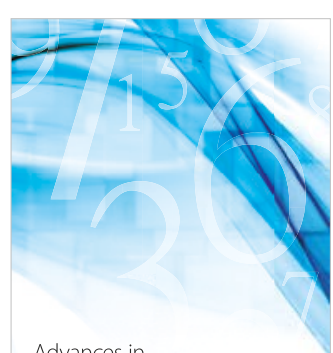

Advances in
Numerical Analysis


Mathematical Problems in Engineering

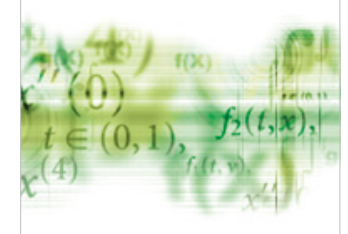

International Journal of

Differential Equations

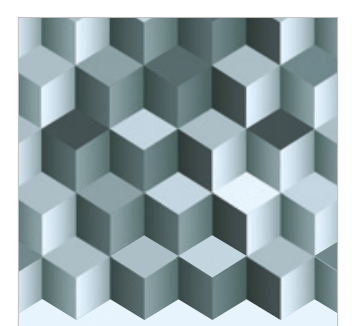

Journal of

Function Spaces



The Scientific

World Journal

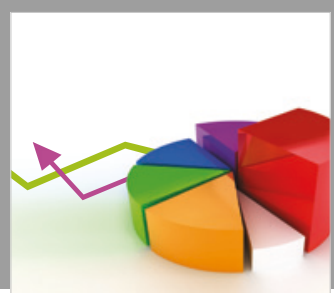

Journal of

Probability and Statistics
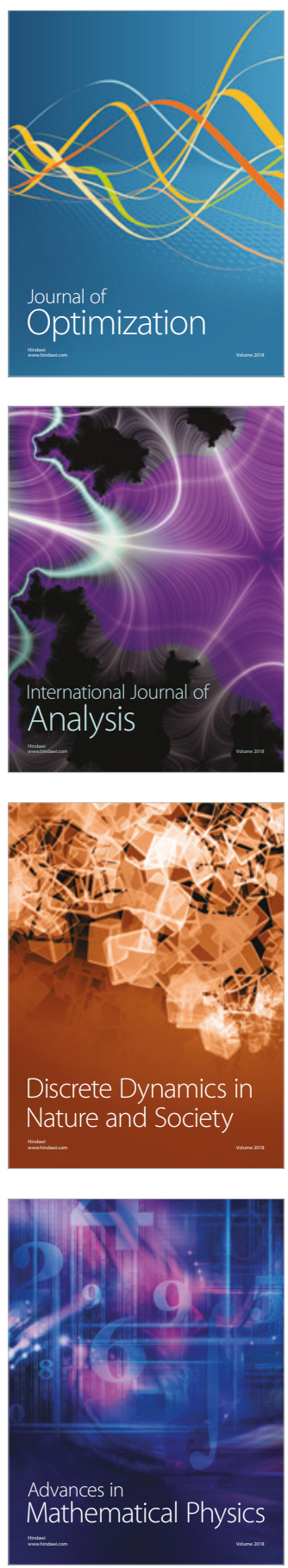